\title{
Recurrence of entire transcendental functions with simple post-singular sets
}

\author{
by \\ Jan-Martin Hemke (Kiel)
}

\begin{abstract}
We study how the orbits of the singularities of the inverse of a meromorphic function determine the dynamics on its Julia set, at least up to a set of (Lebesgue) measure zero. We concentrate on a family of entire transcendental functions with only finitely many singularities of the inverse, counting multiplicity, all of which either escape exponentially fast or are pre-periodic. For these functions we are able to decide whether the function is recurrent or not. In the case that the Julia set is not the entire plane we also obtain estimates for the measure of the Julia set.
\end{abstract}

1. Introduction. One of the main ideas in complex dynamics is to divide the plane into the Fatou set of points where the iterates behave stably, i.e. where they form a normal family, and its complement, the Julia set. By definition the dynamics in the Fatou set is the simplest, and it is very well understood. We are interested in the dynamics of meromorphic functions on their Julia sets. In [6] H. Bock proved the following

THEOREM 1.1 (Bock). For any non-constant meromorphic function defined on the whole complex plane, one of the following two cases holds:

(i) the Julia set of $f$ is the entire plane and for all $A \subset \mathbb{C}$ of positive measure, all $m \in \mathbb{N}$ and almost all $z \in \mathbb{C}$ there are infinitely many $n \in \mathbb{N}$ with $f^{m n}(z) \in A$;

(ii) almost every forward orbit in the Julia set accumulates only on the post-singular set.

Here the post-singular set is the closure of the union of the forward orbits of all singularities of the inverse function, which are the critical and asymptotic values. This result is a generalization of similar results for rational functions, obtained by M. Lyubich [16] and C. McMullen [19].

We introduce some important terms from ergodic theory which are related to the classification above. A meromorphic function is called ergodic

2000 Mathematics Subject Classification: Primary 37F10. 
(with respect to the Lebesgue measure) if any invariant set has full measure or measure zero. It is called recurrent if for every set $A \subset \mathbb{C}$ and almost every point $z \in A$ the forward orbit $O^{+}(z)$ intersects $A$ infinitely many times. It is easy to see that (i) implies recurrence and ergodicity. Case (ii) rules out neither of these two in general. If however $P(f) \neq \widehat{\mathbb{C}}$, it implies non-recurrence.

It is natural to ask which case holds for a given function. Since a nonempty Fatou set always implies (ii), one can restrict oneself to the cases in which the Julia set is the whole complex plane. If it is not, and thus (ii) holds, it would still be interesting to know whether it has positive measure, since otherwise statement (ii) would be trivial.

In the paper mentioned (see also [5]), H. Bock gives sufficient conditions for (i): if $f$ is entire, and the set of singularities of the inverse function is finite and all of them are pre-periodic but not periodic, then (i) is satisfied. The function $f(z)=2 \pi i \exp (z)$ is an example, with the post-singular set consisting of the only asymptotic value, zero, and its image $2 \pi i$. Other conditions concerning this case are given by L. Keen and J. Kotus [14]. Conversely, it was already shown in 1984, independently by M. Rees [24] and M. Lyubich [17], that the function $f(z)=\exp (z)$ is an example for (ii). Here the post-singular set consists of the closure of the forward orbit of the only asymptotic value, zero, which tends to infinity on the real axis. This result was generalized in [12] to functions $f_{\lambda}(z)=\lambda \exp (z)$ with $f_{\lambda}^{n}(0)$ tending to infinity sufficiently fast. M. Urbański and A. Zdunik [28] even showed that the Hausdorff dimension of the remaining set is smaller than 2.

The difference between the dynamics of $\exp (z)$ and $2 \pi i \exp (z)$ is caused by the different behavior of the asymptotic value zero under iteration. One might hope for a classification of the two cases depending on the behavior of the singularities of the inverse. As a first approach, we restrict ourselves to functions with few singularities of the inverse, with simple orbits. As we said, we can neglect all orbits that would imply the existence of a component of the Fatou set, such as periodic critical points or infinite orbits that converge in $\mathbb{C}$. The simplest orbits that remain are pre-periodic or escaping ones. If one considers meromorphic functions with poles, another interesting case is that of singularities which are eventually mapped onto a pole. This case has been studied by B. Skorulski for the tangent family in [27] and for a larger class of functions in his recent thesis.

We are interested in conditions ensuring case (ii). In the third section we prove the rather technical Theorem 3.1, which provides a set of sufficient conditions for this case. The proof applies the method developed by M. Rees to construct a positive measure set of points whose iterates show a "spiral" type behavior: they are eventually mapped close to some asymptotic value, then follow its orbit for a certain number of iterates, coming close to infinity, 
until they are mapped again, and even closer than before, to some asymptotic value etc. These orbits are not dense in $\widehat{\mathbb{C}}$, which implies (ii). Since these orbits accumulate at infinity, the only type of components that could possibly intersect this set are wandering and Baker domains. At least for the various families (e.g. critically finite entire functions) in which those do not occur (see Remark 3.2), we also conclude that the Julia set has positive measure.

In Section 4 we consider functions which may be written in the form $f(z)=\int_{0}^{z} P(t) \exp (Q(t)) d t+c$ with polynomials $P$ and $Q$ and $c \in \mathbb{C}$ such that $Q$ is not constant and $P$ not zero. These functions have at most $\operatorname{deg}(Q)$ asymptotic values and $\operatorname{deg}(P)$ critical points. In the extreme case that all singularities of the inverse are pre-periodic but not periodic, the theorem of H. Bock implies (i). We consider the other extreme case, in which the singularities of the inverse tend to infinity. It turns out that we may neglect the critical values, but have to specify the speed of escape of the asymptotic values. We say that a point $z$ escapes exponentially if $\left|f^{n}(z)\right| \geq \exp \left(\left|f^{n-1}(z)\right|^{\delta}\right)$ for some $\delta>0$ and almost all $n \in \mathbb{N}$. Of course for subsets of $\mathbb{N}$ the term almost all means that the complement is finite and does not refer to the Lebesgue measure. With this terminology Theorem 3.1 yields the following principal result.

Theorem 1.2. Let $P$ and $Q$ be polynomials with $P$ not zero and $Q$ not constant, $c \in \mathbb{C}$ and

$$
f(z):=\int_{0}^{z} P(t) \exp (Q(t)) d t+c .
$$

Suppose that all asymptotic values escape exponentially. Then the Julia set has positive measure and $\omega(z) \subset P(f)$ for almost every $z \in J(f)$. If $\operatorname{deg}(Q) \geq 3$, then meas $(F(f))<\infty$.

Here $\omega(z)$ denotes the $\omega$-limit set that consists of all accumulation points of the sequence $\left(f^{n}(z)\right)$. Conversely, one may ask whether almost every orbit in the Julia set accumulates at every singularity $s$ of $f^{-1}$. It is easy to find examples for which this is not the case if $s$ is a critical value. In order not to accumulate at an asymptotic value $s$, an orbit has to stay out of an entire sector. In other contexts, sets with this property turned out to have measure zero. Thus one may expect that indeed for almost every point $z \in J(f)$ every asymptotic value $s$ is contained in $\omega(z)$. If, however the set of points in the Julia set whose orbits are bounded had positive measure, there would be no reason why these orbits should accumulate at a given asymptotic value. It is not known whether this can actually occur, or similarly, whether the Julia set of a polynomial may have positive measure, which is a well known open question. A positive answer to this question would suggest a negative answer 
to our initial question also for asymptotic values. However, under additional assumptions on the critical values the answer is affirmative. More precisely we get the following.

THeOREM 1.3. Let $f$ be as in Theorem 1.2 and again suppose all its asymptotic values escape exponentially. Suppose that every critical point either also escapes exponentially, is pre-periodic or is contained in an attractive Fatou component. Then $\omega(z)=\overline{O^{+}(A)}$ for almost every point $z \in J(f)$, where $A$ denotes the set of asymptotic values.

We define the multiplicity of an asymptotic value $s$ as the supremum of the set of natural numbers $n$ with the following property: there exists an $\varepsilon_{0}>0$ such that for all $\varepsilon<\varepsilon_{0}$ the set $f^{-1}(B(s, \varepsilon))$ contains at least $n$ unbounded components. Then the functions above have exactly $\operatorname{deg}(Q)$ asymptotic values and $\operatorname{deg}(P)$ critical points, counting multiplicity, and may even be characterized as those entire transcendental functions with this property. This was proved by G. Elfving in [9]. He generalized a method introduced by R. Nevanlinna [21], who showed the same for $\operatorname{deg}(P)=0$. This method is summarized in [22].

THEOREM 1.4 (Elfving). Let $f$ be entire transcendental, with only finitely many singularities of its inverse, counting multiplicity. Then there exist polynomials $P, Q$ and some $c \in \mathbb{C}$ such that

$$
f(z)=\int_{0}^{z} P(t) \exp (Q(t)) d t+c .
$$

For an entire transcendental function $f$ with only finitely many singularities of the inverse, all of which are pre-periodic or escape exponentially, the set $P(f)$ does not accumulate in $\mathbb{C}$, in particular not everywhere in $\mathbb{C}$. Therefore if (ii) is satisfied, the function cannot be recurrent. Thus for this restricted family of functions, the question whether (i) or (ii) is true is equivalent to the question whether $f$ is recurrent or not. As an answer to this question we get the following.

THEOREM 1.5. Let $f$ be entire and transcendental with only a finite number of singularities of its inverse, counting multiplicity, such that all these either escape exponentially or are pre-periodic, but no critical point is periodic. Then $f$ is not recurrent if and only if all asymptotic values escape exponentially.

It is remarkable that the condition in Theorem 1.5 only depends on the asymptotic values. In the last section we discuss applications of Theorem 3.1 to other families, especially transcendental meromorphic functions with rational Schwarzian derivative. 
Acknowledgements. I would like to thank my advisor Walter Bergweiler and the complex dynamics seminar of the CAU-Kiel for constant support and encouragement. Moreover, I owe thanks to Nuria Fagella, Xavi Xarque, the dynamical systems group at the Universidád Autónoma de Barcelona and the Marie Curie Fellowship Association for a productive and marvellous year in Barcelona.

2. Preliminaries. Let $f^{k}$ denote the $k$ th iterate, and $f^{(k)}$ the $k$ th derivative of $f$. Let "meas" denote the Lebesgue measure in the plane, "dist" the Euclidean distance, and "diam" the diameter. For any $r>0$ and $z \in \mathbb{C}$, let $B(z, r)$ denote the open ball of radius $r$ and center $z, B(M, r):=$ $\bigcup_{z \in M} B(z, r)$ for $M \subset \mathbb{C}$ and $D(r):=\mathbb{C} \backslash B(0, r)$. For a square $S$ let $r S$ denote the square with the same center and with $\operatorname{diam}(r S)=r \operatorname{diam}(S)$. For a conformal map $f: D \rightarrow \mathbb{C}$ we call $\sup _{z, w \in D}\left|f^{\prime}(z) / f^{\prime}(w)\right|$ its distortion. We state the well known Koebe distortion theorem as may be found in [23].

Theorem 2.1 (Koebe). Suppose $f: B(0,1) \rightarrow \mathbb{C}$ is conformal with $f(0)=0$ and $f^{\prime}(0)=1$, and $z \in B(0,1)$. Then

$$
\begin{gathered}
\frac{1-|z|}{(1+|z|)^{3}} \leq\left|f^{\prime}(z)\right| \leq \frac{1+|z|}{(1-|z|)^{3}}, \\
\frac{|z|}{(1+|z|)^{2}} \leq|f(z)| \leq \frac{|z|}{(1-|z|)^{2}}, \\
\frac{1-|z|}{1+|z|} \leq\left|z \frac{f^{\prime}(z)}{f(z)}\right| \leq \frac{1+|z|}{1-|z|} .
\end{gathered}
$$

This theorem implies in particular the following corollary, which is known as Koebe's 1/4-Theorem.

COROllary 2.2 (Koebe). Let $f$ be as in Theorem 2.1. Then

$$
B(0,1 / 4) \subset f(B(0,1)) .
$$

It is much easier to show the following property, which will be sufficient for most of our purposes.

Lemma 2.3. Let $f: B\left(z_{0}, r\right) \rightarrow \mathbb{C}$ be holomorphic. Then

$$
B\left(f\left(z_{0}\right), \inf _{z \in B\left(z_{0}, r\right)}\left|f^{\prime}(z)\right| r\right) \subset f\left(B\left(z_{0}, r\right)\right) .
$$

Proof. We can assume that $f$ has no critical points. We consider the straight line segment $\gamma$ from $f\left(z_{0}\right)$ to the closest boundary point of the image. The pre-image of $\gamma$ contains a path $\gamma^{\prime}$ connecting $z_{0}$ with the boundary of $B\left(z_{0}, r\right)$, which is mapped by $f$ one-to-one onto $\gamma$. Since the length of $\gamma^{\prime}$ is at least $r$, the length of $\gamma$ is at least $\inf _{z \in B\left(z_{0}, r\right)}\left|f^{\prime}(z)\right| r$. 
Rather than discs, we will be more interested in the distortion of squares. From Koebe's distortion theorem one can obtain similar estimates for squares. The following lemma will be sufficient for our purpose and follows from Koebe's distortion theorem. However, one could also prove it more directly using normal families.

Lemma 2.4. For any $0<c<1$ there exists a $K_{c}>0$ such that for any holomorphic function which is injective on some square $S$, the distortion of its restriction to $c S$ is bounded by $K_{c}$. Moreover, $K_{c}$ tends to 1 as $c \rightarrow 0$.

The following lemma follows directly from our definition of distortion.

Lemma 2.5. Suppose that the distortion of the conformal map $f$ is bounded by $K$. Let $D$ and $M$ be measurable subsets of its domain of definition with meas $(D)>0$. Then

$$
\frac{\operatorname{meas}(M \cap D)}{\operatorname{meas}(D)} \leq \frac{K^{2} \operatorname{meas}(f(M) \cap f(D))}{\operatorname{meas}(f(D))} .
$$

The term on the left side of (6) is called the density of $M$ in $D$. In the next lemma we state two properties of quasi-squares, which we will frequently use. The proof is simple and may be found in [12].

Lemma 2.6. Let $D$ be a $K$-quasi-square and $\varepsilon>0$. Then

$$
\operatorname{meas}(D) \geq \frac{\operatorname{diam}(D)^{2}}{2 K^{2}}, \quad \operatorname{meas}(D \cap B(\partial D, \varepsilon)) \leq 4 \varepsilon K^{2} \operatorname{diam}(D) .
$$

Here $\partial S$ denotes the boundary of a set $S \subset \mathbb{C}$. Finally, we state a tool which we will frequently use to obtain injectivity of a function on certain sets. It is a corollary of the Monodromy Theorem, to be found in most function theory books, such as [7].

Lemma 2.7. Let $D^{\prime} \subset D \subset \mathbb{C}$ be domains, and $f: D \rightarrow \mathbb{C}$ holomorphic such that all singularities of the inverse of $f$ are contained in the unbounded component of $\mathbb{C} \backslash f\left(D^{\prime}\right)$. Then $f$ is injective on $D^{\prime}$.

To avoid confusion we add the definition of a singularity of $f^{-1}$.

Definition 2.8. Let $D \subset \widehat{\mathbb{C}}$ be a domain, $f: D \rightarrow \widehat{\mathbb{C}}$ be meromorphic and $s \in \widehat{\mathbb{C}}$. Then $s$ is called a singularity of $f^{-1}$ if there exist

- a smooth function $\gamma:[0,1] \rightarrow \widehat{\mathbb{C}}$ with $\gamma(1)=s$;

- a domain $U \subset \widehat{\mathbb{C}}$ with $\gamma([0,1)) \subset U$;

- a branch $\phi$ of the inverse of $f$ on $U$, i.e. $\phi: U \rightarrow D$ meromorphic with $f(\phi(z))=z$,

such that there is no domain $V \subset \widehat{\mathbb{C}}$ with $\gamma([0,1]) \subset V$, and no branch $\psi$ of the inverse of $f$ on $V$ that coincides with $\phi$ on the component of $U \cap V$ containing $\gamma([0,1))$. We denote the set of singularities of $f^{-1}$ by $\operatorname{sing}\left(f^{-1}\right)$. 
Studying the set $A:=\bigcap_{t \in(0,1)} \overline{\phi((t, 1))}$, one can classify these as follows.

Theorem 2.9. Let $D, f$ be as above and $s \in \operatorname{sing}\left(f^{-1}\right)$. Let $\gamma, U$ and $\phi$ be as in Definition 2.8. Then one of the following cases holds.

- There exists $z \in D$ with $f(z)=s$ and $\phi(\gamma(t)) \rightarrow z$ as $t \rightarrow 1$. If neither $z$ nor $s$ coincides with $\infty$ then $f^{\prime}(z)=0$.

- $\operatorname{dist}(\phi(\gamma(t)), \partial D) \rightarrow 0$ as $t \rightarrow 1$.

In the first case $s$ is called a critical value and in the second case an asymptotic value.

In the literature sometimes the closure of the set of singularities of the inverse is denoted by the same name. However if this set is finite, which is the case for all functions we consider, this makes no difference.

It is evident that the pre-image of a neighborhood of an asymptotic value of an entire function must contain an unbounded component. Thus the multiplicity, as defined in the introduction, is always at least 1. It may however be larger and even infinite.

3. Non-recurrence. We follow the ideas used by M. Rees for the exponential function. We obtain a set of points with positive measure, whose orbits are not dense in $\mathbb{C}$, and therefore rule out case (i). This provides a set of sufficient conditions for case (ii). In order to allow a wide application, and hoping for further generalizations, we state our theorem as generally as possible. It is therefore rather technical.

Theorem 3.1. Let $f$ be meromorphic, $A \subset \mathbb{C}$ finite and $G \subset \mathbb{C}$ such that:

(a) there exists $\varepsilon>0$ such that the map

$$
\bar{s}: G \rightarrow A \cup\{0\}, \quad z \mapsto \begin{cases}s & \text { if } \exists s \in A:|f(z)-s| \leq \exp \left(-|z|^{\varepsilon}\right), \\ 0 & \text { if }|f(z)| \geq \exp \left(|z|^{\varepsilon}\right),\end{cases}
$$

is well defined and there are $\delta_{1}, \delta_{2} \in \mathbb{R}$ such that for all $z \in G$,

$$
|z|^{\delta_{1}} \leq\left|\frac{f^{\prime}(z)}{f(z)-\bar{s}(z)}\right| \leq|z|^{\delta_{2}}
$$

(b) there exist $B>1$ and $\beta \in(-\infty, 1)$ such that for every measurable set $D \subset\left\{z: \operatorname{dist}(z, \mathbb{C} \backslash G) \leq 2|z|^{-\delta_{1}}\right\}$,

$$
\operatorname{meas}(D) \leq B \operatorname{diam}(D) \sup _{z \in D}|z|^{\beta}
$$

(c) $f^{m}(s) \rightarrow \infty$ as $m \rightarrow \infty$ and $B\left(f^{m}(s), 2\left|f^{m}(s)\right|^{\tau}\right) \subset G$ for some $\tau>\beta$, almost all $m \in \mathbb{N}$ and all $s \in A$.

Then the set $T(f):=\left\{z: \omega(z) \subset \overline{O^{+}(A)}\right\}$ has positive measure. Further, there exists $M>0$ such that for any square $T_{0} \subset\left\{z: \operatorname{dist}(z, \mathbb{C} \backslash G)>|z|^{-\delta_{1}}\right\}$ 
with $M_{0}:=\inf _{z \in T_{0}}|z|>M$ and $\operatorname{diam}\left(T_{0}\right) \geq M_{0}^{-\delta_{2}}$ we have

$$
\frac{\operatorname{meas}\left(T(f) \cap T_{0}\right)}{\operatorname{meas}\left(T_{0}\right)} \geq 1-\exp \left(-\eta M_{0}^{\varepsilon}\right)
$$

where $\eta:=(\tau-\beta) / \max \{1,2-2 \tau\}>0$.

REMARK 3.2. We would like to know that $T(f) \subset J(f)$. Since the orbits of all points in $T(f)$ accumulate at infinity, the only components that could possibly intersect $T(f)$ are Baker domains and wandering domains. There are various families in which these do not occur. For the family which we consider in the next section, the absence of wandering has been shown by I. N. Baker in [1]. For entire functions with only finitely many singularities of the inverse this has been shown by A. Eremenko and M. Lyubich in [10] and by L. R. Goldberg and L. Keen in [11]. For meromorphic functions with the same property this has been shown by I. N. Baker, J. Kotus and Y. Lü in [4]. The absence of Baker domains has been shown for entire functions with a bounded set of singularities of the inverse by A. Eremenko and M. Lyubich in [10] and for meromorphic functions for which this set is finite by P. J. Rippon and G. M. Stallard in [25]. Moreover in [2] I. N. Baker obtained an upper estimate of the growth of $\left|f^{n}(z)\right|$ for a point $z$ in the Baker domain of an entire function, which is not compatible with the iterated exponential escape; we will find it in the proof below for points escaping to $\infty$ in $T(f)$. Similar estimates implying the same for meromorphic functions have been obtained in [3].

It also makes sense to choose $A=\emptyset$. Then we obtain sufficient conditions for meas $(I(f))>0$, where $I(f)$ denotes the set of escaping points (see 5.1).

Proof of Theorem 3.1. From our conditions (b) and (c) one can deduce that $-\delta_{1}<\beta<\tau<1$. We note that for any $M>0$, taking $B$ sufficiently large allows us to assume that $G \cap B(0, M)=\emptyset$. For all $s \in A$ we define

$$
\begin{aligned}
m_{s} & :=\max \left(\left\{m \in \mathbb{N}: f^{\prime}\left(f^{m-1}(s)\right)=0\right\} \cup\{0\}\right), \\
k_{s} & :=\min \left\{k \in \mathbb{N}:\left(f^{m_{s}}\right)^{(k)}(s) \neq 0\right\} .
\end{aligned}
$$

The distortion constant $K_{c}$ from Lemma 2.4 tends to 1 as $c \rightarrow 0$. Thus for $c>0$ small enough we have $c K_{c} / 4<1$. Since $A$ is finite one can even find $c>0$ such that $k_{s} \arcsin (c K / 4)<\pi$ for all $s \in A$ and $K:=K_{c}$. Suppose that $\delta>0$ is small. In fact, it turns out that $\delta<(\tau-\beta)(1-\tau) /(6-5 \tau-\beta)$ is sufficient for all requirements needed. Similarly choose $M>0$ sufficiently large to satisfy the bounds appearing throughout the proof. For now we only require the following two properties. Firstly, for any $M_{0}>M$ the series $\sum M_{k}$ defined by

$$
M_{k+1}:=\exp \left(\min \left\{1, \frac{1}{2-2 \tau}\right\} M_{k}^{\varepsilon}\right)
$$


tends to infinity fast enough, so that

$$
\prod_{k \in \mathbb{N}}\left(1-\frac{1}{4} M_{k}^{\beta-\tau}\right) \geq 1-M_{1}^{\beta-\tau}
$$

Secondly, there are no critical points in $A_{k}$ for all $k \in \mathbb{N} \cup\{0\}$ where

$$
\begin{aligned}
& A_{k}:=\left(D\left(\frac{1}{2} M_{k+1}^{1 /\left(1+2 \delta_{2}-\delta_{1}+3 \delta\right)}\right) \cap G\right) \\
& \cup \bigcup_{0 \leq l \leq m_{s}}\left(B\left(f^{l}(s), M_{k+1}^{\delta-1}\right) \backslash\left\{f^{l}(s)\right\}\right) \cup \bigcup_{l>m_{s}} B\left(f^{l}(s), a_{k, s, l}\right), \\
& a_{k, s, l}:=\sup \left\{\left|f^{j}(s)\right|^{-\delta_{2}}: j \geq l,\left|f^{j+1}(s)\right| \geq M_{k+1}^{1 /\left(1+2 \delta_{2}-\delta_{1}+3 \delta\right)}\right\} .
\end{aligned}
$$

Of course, at this point we only need to study $A_{0}$ since $A_{k+1} \subset A_{k}$. The set $A_{0}$ does not contain any critical points for $M_{0}$ large enough, since those do not accumulate in $\mathbb{C}$ and, by condition (a), $G$ does not contain any critical points. We note that due to (c) every $s \in A$ escapes in $G$ exponentially fast, so that $a_{k, s, l} \leq\left|f^{l}(s)\right|^{-\delta_{2}}$ for large $l$.

Now let $T_{0}$ and $M_{0}$ be as in the theorem. Let $\mathcal{S}$ be a family of disjoint open squares $S \subset\left\{z: \operatorname{dist}(z, \mathbb{C} \backslash G) \geq|z|^{-\delta_{1}}\right\}$ satisfying

$$
\frac{c}{8}\left(\inf _{z \in c^{-1} S}|z|\right)^{-\delta_{2}} \leq \operatorname{diam}(S) \leq \frac{c}{2}\left(\sup _{z \in c^{-1} S}|z|\right)^{-\delta_{2}},
$$

whose union covers $\left\{z \in G: \operatorname{dist}(z, \mathbb{C} \backslash G) \geq 2|z|^{-\delta_{1}}\right\}$ up to a set of measure zero, such that $\bar{T}_{0}=\bigcup_{S \in X} \bar{S}$ for some finite $X \subset \mathcal{S}$. A picture of this could look like Figure 1. We can get this by covering the whole plane with open squares of a constant diameter, beginning with $T_{0}$, cutting these into four until their diameter satisfies the upper bound, and discarding those
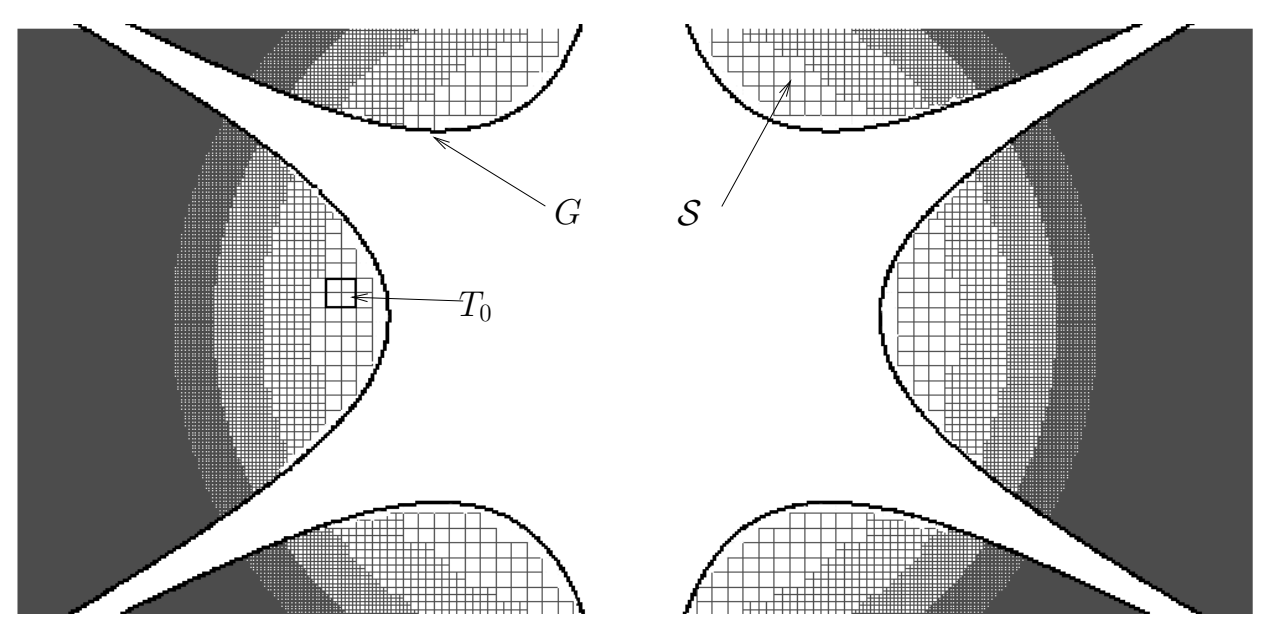

Fig. 1. The family $\mathcal{S}$ 
intersecting $\left\{z: \operatorname{dist}(z, \mathbb{C} \backslash G) \leq|z|^{-\delta_{1}} / 2\right\}$. For $M$ large enough and for $G \cap B(0, M)=\emptyset$ our squares also satisfy the lower bound. We prove the measure estimate in the theorem for all elements of $\mathcal{S}$ including the ones in $X$. This implies this estimate also for $T_{0}$. Thus we proceed with an element of $\mathcal{S}$, which we again call $T_{0}$.

From the estimates of condition (a) one can show that if $\left|z_{0}\right|$ is large enough and $B\left(z_{0},\left|z_{0}\right|^{-\delta_{1}}\right) \subset G$ then

$$
f \text { is injective on } B\left(z_{0},\left|z_{0}\right|^{-\delta_{2}} / 4\right) \text {. }
$$

To see this we use Lemma 2.7 and show first that

$$
f\left(B\left(z_{0},\left|z_{0}\right|^{-\delta_{2}} / 4\right)\right) \subset B\left(f\left(z_{0}\right), 3\left|f\left(z_{0}\right)-\bar{s}\left(z_{0}\right)\right| / 8\right) \subset f\left(B\left(z_{0},\left|z_{0}\right|^{-\delta_{1}}\right)\right) .
$$

Indeed, if the first inclusion were false, we would find $z \in B\left(z_{0},\left|z_{0}\right|^{-\delta_{2}} / 4\right)$ with

$$
\left|f(z)-f\left(z_{0}\right)\right| \geq 3\left|f\left(z_{0}\right)-\bar{s}\left(z_{0}\right)\right| / 8 .
$$

We choose $\left|z-z_{0}\right|$ minimal with this property, so that for $x$ on the segment $\left(z, z_{0}\right):=\left\{(1-t) z+t z_{0}: 0<t<1\right\}$ we have

$$
\left|f(x)-\bar{s}\left(z_{0}\right)\right| \leq\left|f(x)-f\left(z_{0}\right)\right|+\left|f\left(z_{0}\right)-\bar{s}\left(z_{0}\right)\right| \leq \frac{11}{8}\left|f\left(z_{0}\right)-\bar{s}\left(z_{0}\right)\right| .
$$

The mean value theorem provides $x \in\left(z, z_{0}\right)$ with

$$
\left|f^{\prime}(x)\right| \geq \frac{\left|f(z)-f\left(z_{0}\right)\right|}{\left|z-z_{0}\right|} \geq \frac{3}{2}\left|f\left(z_{0}\right)-\bar{s}\left(z_{0}\right)\right|\left|z_{0}\right|^{\delta_{2}} \geq \frac{12}{11}\left|f(x)-\bar{s}\left(z_{0}\right)\right|\left|z_{0}\right|^{\delta_{2}} .
$$

This contradicts (a), since for $z_{0}$ large enough $\left|x-z_{0}\right| \leq\left|z_{0}\right|^{-\delta_{2}}$ is very small, so that $\bar{s}(x)=\bar{s}\left(z_{0}\right)$ and $|x|>\left(\frac{11}{12}\right)^{-\delta_{2}}\left|z_{0}\right|$.

The second inclusion follows from the fact that there are no critical points in $B\left(z_{0},\left|z_{0}\right|^{-\delta_{1}}\right) \subset G$. Thus we may extend the branch of $f^{-1}$, mapping $f\left(z_{0}\right)$ to $z_{0}$ along any path in $B\left(f\left(z_{0}\right), 3\left|f\left(z_{0}\right)-\bar{s}\left(z_{0}\right)\right| / 8\right)$ as long as the image stays in $B\left(z_{0},\left|z_{0}\right|^{-\delta_{1}}\right)$. As above the mean value theorem and condition (a) ensure this, since for a given $x \in B\left(z_{0},\left|z_{0}\right|^{-\delta_{1}}\right)$ with $f(x) \in$ $B\left(f\left(z_{0}\right), 3\left|f\left(z_{0}\right)-\bar{s}\left(z_{0}\right)\right| / 8\right)$ it follows that $\left|f(x)-\bar{s}\left(z_{0}\right)\right| \geq 5\left|f\left(z_{0}\right)-\bar{s}\left(z_{0}\right)\right| / 8$, so that

$$
\left|f^{\prime}(x)\right| \geq\left|f(x)-\bar{s}\left(z_{0}\right)\right||x|^{\delta_{1}} \geq \frac{1}{2}\left|f\left(z_{0}\right)-\bar{s}\left(z_{0}\right)\right|\left|z_{0}\right|^{\delta_{1}} .
$$

This implies that the image of any path in $B\left(f\left(z_{0}\right), 3\left|f\left(z_{0}\right)-\bar{s}\left(z_{0}\right)\right| / 8\right)$ in fact stays inside $B\left(z_{0}, 3\left|z_{0}\right|^{-\delta_{1}} / 4\right)$. From this it follows that $f$ is injective on $B\left(z_{0},\left|z_{0}\right|^{-\delta_{2}} / 4\right)$, as claimed. Together with (9) this implies that the distortion of $f$ on any $S \in \mathcal{S}$ is bounded by $K$.

Starting with $F_{0}:=\left\{T_{0}\right\}$ and $n_{0}\left(T_{0}\right):=0$, we will define for every $k \in \mathbb{N}$ a family $\mathcal{F}_{k}$ of disjoint simply connected domains and functions $n_{k}: \mathcal{F}_{k} \rightarrow \mathbb{N}$ such that the sets $T_{k}:=\bigcup \mathcal{F}_{k}=\bigcup_{F \in \mathcal{F}_{k}} F$ form a decreasing sequence with the following properties. For every $U \in \mathcal{F}_{k}$ and the corresponding $V \in \mathcal{F}_{k-1}$ 
with $U \subset V$ :

(i) $D\left(M_{k}\right) \supset f^{n_{k}(U)}(U) \in \mathcal{S}$ and $c^{-1} f^{n_{k}(U)}(U) \subset f^{n_{k}(U)}(V)$;

(ii) $f^{j}(V) \subset A_{k}$ for every $n_{k-1}(V)<j<n_{k}(U)$;

(iii) $\operatorname{meas}\left(V \cap \bigcup \mathcal{F}_{k}\right) \geq\left(1-\frac{1}{4} M_{k}^{\beta-\tau}\right) \operatorname{meas}(V)$.

Condition (ii) implies that $\omega(z) \subset \bigcap_{k \in \mathbb{N}} A_{k}=O^{+}(A) \cup\{\infty\}$ for all $z \in T:=\bigcap_{k \in \mathbb{N}} T_{k}$. Having (iii) for each component of $T_{k}$, that is, the elements of $\mathcal{F}_{k}$, implies that meas $\left(T_{k}\right)>\left(1-\frac{1}{4} M_{k}^{\beta-\tau}\right)$ meas $\left(T_{k-1}\right)$, which, together with the exponential growth of $M_{k}$, guarantees that

$$
\operatorname{meas}(T) \geq\left(\prod_{k=1}^{\infty}\left(1-\frac{1}{4} M_{k}^{\beta-\tau}\right)\right) \operatorname{meas}\left(T_{0}\right) .
$$

Together with (8) this implies the measure estimate from Theorem 3.1.

It remains to construct the sequences. We will do that inductively. Since the starting step of the induction works just as any other step, we do not consider it separately. We assume the existence of appropriate $\mathcal{F}_{k}$ and $n_{k}$ for some $k \in \mathbb{N}$. Let $U \in \mathcal{F}_{k}$. Then $S:=f^{n_{k}(U)}(U) \in \mathcal{S}$. Due to condition (ii) and the fact that there are no critical points in $A_{0}$, one can extend the inverse of $f^{n_{k}(U)} \mid U$ to $c^{-1} S$ and its distortion on $S$ is bounded by $K$. Furthermore, $S \subset D\left(M_{k}\right)$ so that we can consider the following cases separately.

Case 1: $f(S) \subset D\left(M_{k+1}\right)$. We define

$$
\mathcal{F}:=\left\{R \in \mathcal{S}: c^{-1} R \subset f(S)\right\}, \quad \mathcal{F}_{U}:=\left\{\left(f^{n_{k}(U)+1} \mid U\right)^{-1}(R): R \in \mathcal{F}\right\}
$$

and $n_{k+1}(V):=n_{k}(U)+1$ for all $W \in \mathcal{F}_{U}$. See Figure 2. Then (for $\mathcal{F}_{U}$ in place of $\mathcal{F}_{k+1}$ ) property (i) holds by definition, while property (ii) is trivial. Since $f \mid S$ is injective and its distortion is bounded by $K, f(S)$ is a

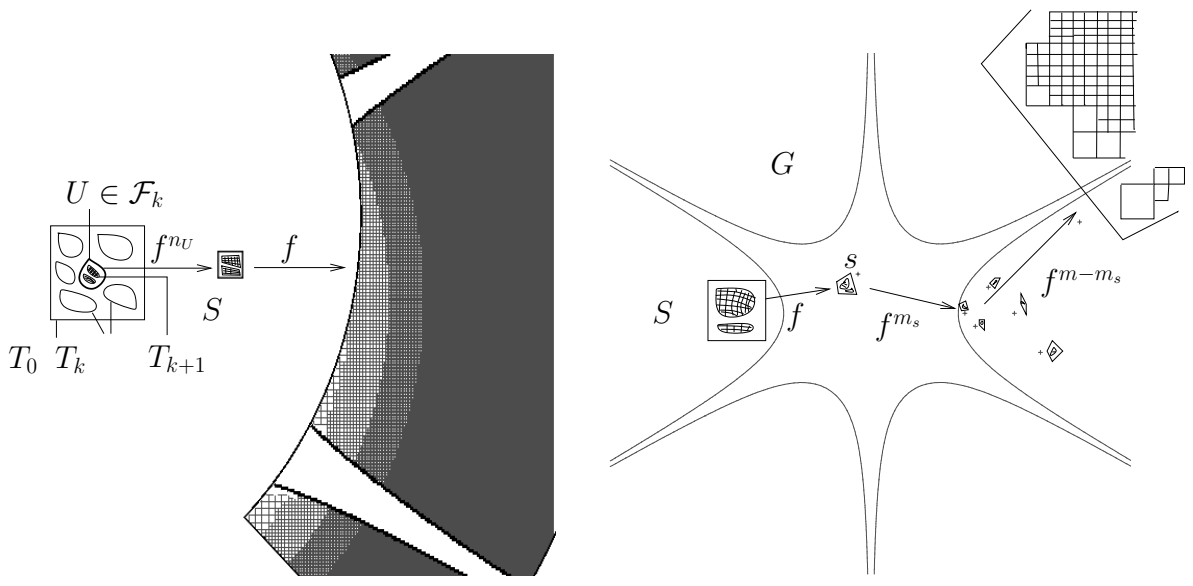

Fig. 2. Models for the construction in both cases 
$K$-quasi-square with

$$
\operatorname{diam}(f(S)) \geq \frac{1}{\sqrt{2}} \operatorname{diam}(S) \inf _{z \in S}\left|f^{\prime}(z)\right| \geq \sup _{z \in S}|f(z)|^{1-\delta}
$$

for $M_{0}$ large enough. Here the last inequality holds since the term $\left|f^{\prime}(z)\right|$ is, due to (a), of magnitude $|f(z)| \geq \exp \left(|z|^{\varepsilon}\right)$. This is far larger than all other factors that appear, so they may be absorbed by $|f(z)|^{\delta}$. Also the infimum may be substituted by the supremum, since the distortion is bounded by $K$.

By definition of $\mathcal{S}$ and $\mathcal{F}$, the set $f(S) \backslash \bigcup \mathcal{F}$ is contained in the union of $\partial \bigcup \mathcal{S}$, which has measure zero, and small neighborhoods of $\partial f(S)$ and $\mathbb{C} \backslash G$. More precisely, we have

$$
\begin{aligned}
\operatorname{meas}(f(S) \backslash \bigcup \mathcal{F}) \leq & \operatorname{meas}\left(\left\{z \in f(S): \operatorname{dist}(z, \partial f(S)) \leq|z|^{-\delta_{2}}\right\}\right) \\
& +\operatorname{meas}\left(\left\{z \in f(S): \operatorname{dist}(z, \mathbb{C} \backslash G) \leq 2|z|^{-\delta_{1}}\right\}\right) .
\end{aligned}
$$

With condition (b) we can control the second term on the right by

$$
\operatorname{meas}\left(\left\{z \in f(S): \operatorname{dist}(z, \mathbb{C} \backslash f(S)) \leq 2|z|^{-\delta_{1}}\right\}\right) \leq B \operatorname{diam}(f(S)) \sup _{z \in f(S)}|z|^{\beta} .
$$

Since $f(S)$ is a $K$-quasi-square, the measure of an $r$-neighborhood of the boundary of $f(S)$ is, due to $(7)$, at most $4 r K^{2} \operatorname{diam}(f(S))$. Also the measure of $f(S)$ is bounded by $\operatorname{diam}(f(S))^{2} / 2 K^{2}$. Since the set in the first term on the right in (12) is contained in the $\sup _{z \in f(S)}|z|^{-\delta_{2}}$-neighborhood of $\partial f(S)$ and we know that $-\delta_{2}<-\delta_{1}<\beta$, we deduce, using (11), that

$$
\begin{aligned}
\frac{\operatorname{meas}(f(S) \backslash \bigcup \mathcal{F})}{\operatorname{meas}(f(S))} & \leq \frac{2 K^{2}\left(4 K^{2} \sup _{z \in f(S)}|z|^{-\delta_{2}}+B \sup _{z \in f(S)}|z|^{\beta}\right)}{\operatorname{diam}(f(S))} \\
& \leq 8 B K^{4} \sup _{z \in f(S)}|z|^{\beta+\delta-1} \leq 8 B K^{4} M_{k+1}^{\beta+\delta-1}
\end{aligned}
$$

for $M$ large enough. As mentioned, the distortions of $f^{n_{k}(U)} \mid U$ and $f \mid S$ are bounded by $K$. Therefore the distortion of $f^{n_{k+1}(V)} \mid U$ is bounded by $K^{2}$ and we get

$$
\frac{\operatorname{meas}\left(U \backslash \bigcup \mathcal{F}_{U}\right)}{\operatorname{meas}(U)} \leq \frac{K^{4} \operatorname{meas}(f(S) \backslash \bigcup \mathcal{F})}{\operatorname{meas}(f(S))},
$$

which, together with (13), implies property (iii) for $\delta$ small and $M$ large enough.

CASE 2: $f(S) \subset B\left(s, M_{k+1}^{-1}\right)$ for some $s \in A$. We will study the behavior of a certain number of iterates of $f$ on $S$. See also Figure 2. We begin with the first iterate. Let $w$ be the center of $S$. For $z \in S$, (9) implies $|z-w| \leq(c / 4)|w|^{-\delta_{2}}$ and (a) implies $\left|f^{\prime}(w)\right| \leq|f(w)-s||w|^{\delta_{2}}$. The mean value theorem provides $x \in[z, w]$ with

$$
|f(z)-f(w)| \leq\left|f^{\prime}(x)\right||z-w| \leq K\left|f^{\prime}(w)\right||z-w| \leq \frac{c}{4} K|f(w)-s| .
$$


Thus we know that $f(S)$ is contained in

$$
B(s,(1+c K / 4)|f(w)-s|) \backslash B(s,(1-c K / 4)|f(w)-s|) .
$$

Since $S$ contains the disc $B\left(w, c / 16 \sqrt{2}|w|^{\delta_{2}}\right)$, (5) together with (a) implies that

$$
B\left(f(w), \frac{c|f(w)-s|}{16 \sqrt{2} K|w|^{\delta_{2}-\delta_{1}}}\right) \subset f(S) .
$$

Next we consider those iterates in which we cannot avoid critical points. We do this in terms of the power series

$$
f^{m_{s}}(z)=f^{m_{s}}(s)+\left(f^{m_{s}}\right)^{\left(k_{s}\right)}(s)(z-s)^{k_{s}}+O\left((z-s)^{k_{s}+1}\right) .
$$

This provides good estimates for $f^{m_{s}}$ and its derivative if $|z-s|$ is very small, which is the case for $z \in f(S)$ since $|f(w)-s| \leq \exp \left(-|w|^{\varepsilon}\right)$. The only purpose of our choice of $c$ was to ensure that the diameter of $f(S)$ is small enough to guarantee that $f^{m_{s}}(s)$ lies in the unbounded component of $\mathbb{C} \backslash f^{m_{s}+1}(S)$. The reader may prefer to convince himself that this goal is achievable by choosing $c$ sufficiently small, instead of checking that our concrete choice above is sufficient. Thus Lemma 2.7 implies that $f^{m_{s}}$ is injective on $f(S)$. The ratio of the outer and inner radii of the annulus in $(14)$ is

$$
C:=\frac{1+c K / 4}{1-c K / 4}
$$

Thus the image lies in an annulus whose ratio of radii is very close to $C^{k_{s}}$ and the distortion is bounded by any constant greater than $C^{k_{s}-1}$, say $C^{k_{s}}$. Using the factor $1 \pm c K / 4$ for the error term of the power series we can deduce from (14) and (15) that

$$
f^{m_{s}+1}(S) \supset B\left(f^{m_{s}+1}(w),\left|\frac{k_{s}\left(f^{m_{s}}\right)^{\left(k_{s}\right)}(s)(1-c K / 4)^{k_{s}}(f(w)-s)^{k_{s}}}{16 \sqrt{2} K|w|^{\delta_{2}-\delta_{1}}}\right|\right)
$$

and

$$
f^{m_{s}+1}(S) \subset B\left(f^{m_{s}}(s),\left|\left(f^{m_{s}}\right)^{\left(k_{s}\right)}(s)(1+c K / 4)^{k_{s}+1}(f(w)-s)^{k_{s}}\right|\right) .
$$

Here no term except $|f(w)-s|$ depends on $k$. One could get similar estimates for $1 \leq l \leq m_{s}+1$; they imply

$$
f^{l}(S) \subset B\left(f^{l-1}(s), M_{k+1}^{\delta-1}\right) \backslash\left\{f^{l-1}(s)\right\}
$$

for $M$ large enough. This implies that $f^{l}(S)$ is contained in $A_{k}$ or, more precisely, in the middle term of its definition.

Next we consider the maximal number of iterates for which we can ensure injectivity and bounded distortion. By (c) the set $B\left(f^{m}(s), 16\left|f^{m}(s)\right|^{-\left(\delta_{2}+\delta\right)}\right)$ is contained in $\left\{z: \operatorname{dist}(z, \mathbb{C} \backslash G) \geq|z|^{-\delta_{1}}\right\} \cap B\left(f^{m}(s),\left|f^{m}(s)\right|^{-\delta_{2}}\right)$ for $m$ large enough. For $m$ large, $\left|f^{m}(s)\right|^{\delta}>64$ so that, due to (10), $f$ restricted to this 
set is injective. By Koebe's 1/4-theorem we get

$$
\begin{aligned}
f\left(B\left(f^{m}(s), 8\left|f^{m}(s)\right|^{-\left(\delta_{2}+\delta\right)}\right)\right) & \supset B\left(f^{m+1}(s), \frac{2\left|f^{\prime}\left(f^{m}\right)(s)\right|}{\left|f^{m}(s)\right|^{\delta_{2}+\delta}}\right) \\
& \supset B\left(f^{m+1}(s), \frac{2\left|f^{m+1}(s)\right|}{\left|f^{m}(s)\right|^{\delta_{2}-\delta_{1}+\delta}}\right) .
\end{aligned}
$$

Here the last inclusion follows from (a) and (c). By (c) we know that $f^{m}(s)$ escapes to $\infty$ in $G$. For $z \in G$, (a) implies $\left|f^{\prime}(z)\right| \geq|f(z)||z|^{\delta_{1}}$. Thus $\left|f^{\prime}\left(f^{m}(s)\right)\right| \rightarrow \infty$ as $m \rightarrow \infty$. Hence for $m$ large enough, $m_{s} \leq l \leq m$ and $r>0$ small we know that $f^{m-l}$ is expanding on $B\left(f^{l}(s), r\right)$. Consequently, the component of $\left(f^{m-l}\right)^{-1}\left(B\left(f^{m}(s), 8\left|f^{m}(s)\right|^{-\left(\delta_{2}+\delta\right)}\right)\right)$ containing $f^{l}(s)$ is contained in $B\left(f^{l}(s), 8\left|f^{m}(s)\right|^{-\left(\delta_{2}+\delta\right)}\right)$, which does not contain critical points. This allows us to extend the inverse $g$ of $f^{m-m_{s}+1}$, mapping $f^{m+1}(s)$ to $f^{m_{s}}(s)$, to $B\left(f^{m+1}(s), 2\left|f^{m+1}(s)\right| /\left|f^{m}(s)\right|^{\delta_{2}-\delta_{1}+\delta}\right)$. Thus the distortion on half the ball is bounded by some constant $\widetilde{K}$. One could use Lemma 2.4 to obtain $\widetilde{K}=K_{1 / \sqrt{2}}$ or the original distortion Theorem 2.1 to obtain $\widetilde{K}=81$. In any case we get

$$
\begin{aligned}
& \operatorname{dist}\left(f^{m_{s}}(s), \partial g\left(B\left(f^{m+1}(s),\left|f^{m+1}(s)\right| /\left|f^{m}(s)\right|^{\delta_{2}-\delta_{1}+\delta}\right)\right)\right) \\
& \geq \frac{\left|f^{m+1}(s)\right|}{\left|f^{m}(s)\right|^{\delta_{2}-\delta_{1}+\delta}} \inf _{z \in B\left(f^{m+1}(s),\left|f^{m+1}(s)\right| /\left|f^{m}(s)\right|^{\delta_{2}-\delta_{1}+\delta}\right)}\left|g^{\prime}(z)\right| \\
& \geq \frac{\left|f^{m+1}(s)\right|}{\widetilde{K}\left|f^{m}(s)\right|^{\delta_{2}-\delta_{1}+\delta}}\left|g^{\prime}\left(f^{m+1}(s)\right)\right| \\
& =\frac{\left|f^{m+1}(s)\right|}{\widetilde{K}\left|f^{m}(s)\right|^{\delta_{2}-\delta_{1}+\delta}\left|\left(f^{m-m_{s}+1}\right)^{\prime}\left(f^{m_{s}}(s)\right)\right|} \\
& =\frac{\left|f^{m+1}(s)\right|}{\widetilde{K}\left|f^{m}(s)\right|^{\delta_{2}-\delta_{1}+\delta} \prod_{i=m_{s}}^{m}\left|f^{\prime}\left(f^{i}(s)\right)\right|} \\
& \geq \frac{1}{\widetilde{K}\left|f^{m}(s)\right|^{1+2 \delta_{2}-\delta_{1}+\delta}\left|f^{m_{s}}(s)\right|^{\delta_{2}} \prod_{i=m_{s}+1}^{m-1}\left|f^{i}(s)\right|^{1+\delta_{2}}} \\
& \geq\left|f^{m}(s)\right|^{-\left(1+2 \delta_{2}-\delta_{1}+2 \delta\right)}
\end{aligned}
$$

for large $m$. We define $m$ as the greatest natural number that satisfies

$$
\left|f^{m-1}(s)\right|^{-\left(1+2 \delta_{2}-\delta_{1}+2 \delta\right)} \geq\left|\left(f^{m_{s}}\right)^{\left(k_{s}\right)}(s)\right|(1+c K / 4)^{k_{s}+1}|f(w)-s|^{k_{s}} .
$$

We note that $m \rightarrow \infty$ as $|f(w)-s| \rightarrow 0$. Thus we can guarantee that $m$ is large by choosing $M$ large. From the choice of $m$ together with estimate (16) we can deduce that $f^{m_{s}+1}(S) \subseteq g\left(B\left(f^{m}(s),\left|f^{m}(s)\right| /\left|f^{m-1}(s)\right|^{\delta_{2}-\delta_{1}+\delta}\right)\right)$. Thus $f^{m-m_{s}}$ restricted to $f^{m_{s}+1}(S)$ is injective, its distortion is bounded 
by $\widetilde{K}$, and

$$
f^{m+1}(S) \subset B\left(f^{m}(s), \frac{\left|f^{m}(s)\right|}{\left|f^{m-1}(s)\right|^{\delta_{2}-\delta_{1}+\delta}}\right) \subset B\left(f^{m}(s), \frac{\left|f^{m}(s)\right|}{2}\right) .
$$

The choice of maximal $m$ guarantees that

$$
\left|f^{m}(s)\right| \geq|f(w)-s|^{-k_{s} /\left(1+2 \delta_{2}-\delta_{1}+3 \delta\right)}
$$

for $M_{0}$ large enough. Together with the discussion below (18), this implies that $f^{k}(S)$ is contained in $A_{k}$ for $m_{s}+1 \leq k \leq m+1$. The exponential growth of $\left|f^{m}(s)\right|$ and our estimates for $\left|f^{\prime}\right|$ imply that

$$
\left|f^{m}(s)\right|^{1-\alpha} \leq\left|\left(f^{m-k}\right)^{\prime}\left(f^{k}(s)\right)\right| \leq\left|f^{m}(s)\right|^{1+\alpha}
$$

for any $\alpha>0$ and any natural numbers $k<m$ with $m$ large enough. This allows us to cancel the smaller factors, transferring (16) and (17) by bounded distortion of $f^{m-m_{s}}$ to

$$
B\left(f^{m+1}(w),\left|f^{m}(s)\right|^{1-\delta}|f(w)-s|^{k_{s}}\right) \subset f^{m+1}(S)
$$

and

$$
f^{m+1}(S) \subset B\left(f^{m}(s),\left|f^{m}(s)\right|^{1+\delta}|f(w)-s|^{k_{s}}\right) .
$$

Again we distinguish between two cases.

CASE 2.1: We have

$$
\left|f^{m}(s)\right| \geq|f(w)-s|^{-k_{s} /(1-\tau+\delta)} .
$$

This is stronger than (20) and from (a) we get

$$
\left|f^{m}(s)\right| \geq \exp \left(\frac{k_{s}}{1-\tau+\delta} M_{k}^{\varepsilon}\right),
$$

which together with (19) implies that $f^{m+1}(S) \subset D\left(M_{k+1}\right)$ for $\delta$ sufficiently small. We define

$\mathcal{F}:=\left\{R \in \mathcal{S}: c^{-1} R \subset f^{m+1}(S)\right\}, \quad \mathcal{F}_{U}:=\left\{\left(f^{n_{k}(U)+m+1} \mid U\right)^{-1}(R): R \in \mathcal{F}\right\}$ and $n_{k+1}(V):=n_{k}(U)+m+1$ for all $V \in \mathcal{F}_{U}$. Again properties (i) and (ii) follow by definition. The distortion of $f \mid S$ is bounded by $K$, while that of $f^{m_{s}} \mid f(S)$ is bounded by $C^{k_{s}}$, and the distortion of $f^{m-m_{s}} \mid f^{m_{s}+1}(S)$ is bounded by $\widetilde{K}$. Thus the distortion of $f^{m+1} \mid S$ is bounded by $\widetilde{K} K C^{k_{s}}$. Therefore $f^{m+1}(S)$ is a $\widetilde{K} K C^{k_{s}}$-quasi-square. For $M$ large enough, (23) together with (21) implies that

$$
\operatorname{diam}\left(f^{m+1}(S)\right) \geq\left|f^{m}(s)\right|^{\tau-2 \delta} \geq \sup _{z \in f^{m+1}(S)}|z|^{\tau-3 \delta} .
$$

As in Case 1 the measure of the set $f^{m+1}(S) \backslash \cup \mathcal{F}$ is bounded by the measure of $\left\{z: \operatorname{dist}(z, \mathbb{C} \backslash G) \leq 2|z|^{-\delta_{1}}\right\}$, which itself is, due to condition (b), bounded by $B \operatorname{diam}\left(f^{m+1}(S)\right) \sup _{z \in f^{m+1}(S)}|z|^{\beta}$ plus meas $\left(\left\{z \in f^{m+1}(S)\right.\right.$ : $\left.\left.\operatorname{dist}\left(z, \partial f^{m+1}(S)\right) \leq|z|^{-\delta_{2}}\right\}\right)$, which in turn is, by (7), bounded above by 
$4 \widetilde{K}^{2} K^{2} C^{2 k_{s}} \operatorname{diam}\left(f^{m+1}(S)\right) \sup _{f^{m+1}(S)}|z|^{-\delta_{2}}$. Again using (7) we find that the measure of $f^{m+1}(S)$ is bounded below by $\operatorname{diam}\left(f^{m+1}(S)\right)^{2} / 2 \widetilde{K}^{2} K^{2} C^{2 k_{s}}$. Using $-\delta_{2}<\beta$ and (25) we can deduce from the above that

$$
\begin{aligned}
\frac{\operatorname{meas}\left(f^{m+1}(S) \backslash \bigcup \mathcal{F}\right)}{\operatorname{meas}\left(f^{m+1}(S)\right)} & \leq 5 B \widetilde{K}^{2} K^{2} C^{2 k_{s}} \sup _{z \in f^{m+1}(S)}|z|^{\beta+3 \delta-\tau} \\
& \leq \frac{M_{k+1}^{\beta-\tau}}{4 \widetilde{K}^{2} K^{4} C^{2 k_{s}}}
\end{aligned}
$$

where the last inequality holds due to (19) and (24) for $M$ large and $\delta$ small enough. The distortion $f^{n_{k}(U)} \mid U$ is bounded by $K$. Thus (26) together with (6) implies (iii).

CASE 2.2: $\left|f^{m}(s)\right|<|f(w)-s|^{-k_{s} /(1-\tau+\delta)}$. From (22) we get

$$
f^{m+1}(S) \subset B\left(f^{m}(s),\left|f^{m}(s)\right|^{1+\delta}|f(w)-s|^{k_{s}}\right) \subset B\left(f^{m}(s),\left|f^{m}(s)\right|^{\tau}\right),
$$

which, due to condition (c), is contained in $\left\{z: \operatorname{dist}(z, \mathbb{C} \backslash G) \geq|z|^{-\delta_{1}}\right\}$.

We distinguish between two more cases:

CASE 2.2.1: $\operatorname{diam}\left(f^{m+1}(S)\right)<(c / 4)\left|f^{m}(s)\right|^{-\delta_{2}}$. Then due to (10), $f \mid f^{m+1}(S)$ is injective and its distortion is bounded by $K$. We define

$$
\mathcal{F}_{U}:=\left\{\left(f^{n_{k}(U)+m+2} \mid U\right)^{-1}(T): T \in \mathcal{S}, c^{-1} T \subset f^{m+2}(S)\right\}
$$

and $n_{k+1}(V):=n_{k}(U)+m+2$. Then properties (i) and (ii) are again satisfied by definition. The bounds of the distortion of $f\left|S, f^{m_{s}}\right| f(S)$ and $f^{m-m_{s}} \mid f^{m_{s}+1}(S)$ are as above. Then $f^{m+2}(S)$ is a $\widetilde{K} K^{2} C^{k_{s}}$-quasi-square and, from (21), it follows that

$$
\operatorname{diam}\left(f^{m+2}(S)\right) \geq\left|f^{m}(s)\right|^{1-\delta}|f(w)-s|^{k_{s}} \inf _{z \in f^{m+1}(S)}\left|f^{\prime}(z)\right| \geq \sup _{z \in f^{m+2}(S)}|z|^{1-\delta} .
$$

Here the last inequality follows for $M$ large enough, since for $z \in f^{m+1}(S)$, the magnitude of $|f(z)|$, and given condition (a), also that of $\left|f^{\prime}(z)\right|$, is $\exp \left(\left|f^{m}(s)\right|^{\varepsilon}\right)$. By $(20)$, this is far larger than the other factors. From condition (b) and (7) we get as before

$$
\begin{aligned}
& \frac{\operatorname{meas}\left(f^{m+2}(S) \backslash\right.}{\operatorname{meas}\left(f^{m+2}(S)\right)}\left.\mathrm{c}_{R \in \mathcal{S}, c^{-1} R \subset f^{m+2}(S)} R\right) \\
& \leq 5 B \widetilde{K}^{2} K^{4} C^{2 k_{s}} \sup _{z \in f^{m+2}(S)}|z|^{\beta+\delta-1} \\
& \leq \exp \left(\left(\frac{1}{2} \exp \left(\frac{M_{k}^{\varepsilon}}{1+2 \delta_{2}-\delta_{1}+3 \delta}\right)\right)^{\varepsilon}\right)^{\beta+2 \delta-1}
\end{aligned}
$$


Here the last inequality follows by (19) and (20). Again the distortion estimates above and (6) imply that

$$
\frac{\operatorname{meas}\left(U \backslash \mathcal{F}_{U}\right)}{\operatorname{meas}(U)} \leq \frac{\widetilde{K}^{2} K^{6} C^{2 k_{s}} \operatorname{meas}\left(f^{m+2}(S) \backslash \bigcup_{T \in \mathcal{S}, c^{-1} T \subset f^{m+2}(S)} T\right)}{\operatorname{meas}\left(f^{m+2}(S)\right)} .
$$

Together with estimate (28) this is far stronger than condition (iii).

CASE 2.2.2: $\operatorname{diam}\left(f^{m+1}(S)\right) \geq(c / 4)\left|f^{m}(s)\right|^{-\delta_{2}}$. We consider a family $\mathcal{F}$ of disjoint open squares $R \subset f^{m+1}(S)$ with diameter $\exp \left(-\left|f^{m}(s)\right|^{\varepsilon / 2}\right)$ such that $\mathcal{F}$ covers all of $f^{m+1}(S)$ except a set of measure zero and an $\exp \left(-\left|f^{m}(s)\right|^{\varepsilon / 2}\right)$-neighborhood of the boundary. As $f^{m+1}(S)$ is a $\widetilde{K} K C^{k_{s_{-}}}$ quasi-square, (7) implies that

$$
\begin{aligned}
\operatorname{meas}\left(f^{m+1}(S) \backslash\right. & \bigcup \mathcal{F}) \\
& \leq 4 \widetilde{K}^{2} K^{2} C^{2 k_{s}} \exp \left(-\left|f^{m}(s)\right|^{\varepsilon / 2}\right) \operatorname{diam}\left(f^{m+1}(S)\right) \\
& \leq \exp \left(-\left|f^{m}(s) / 2\right|^{\varepsilon / 2}\right)
\end{aligned}
$$

for $M$ large enough, since, by (27), again one factor, namely $\exp \left(\left|f^{m}(s)\right|^{\varepsilon / 2}\right)$, dominates all others. We define $\mathcal{F}_{U}$ as

$$
\begin{aligned}
& \left\{\left(\left(f^{n_{k}(U)+m+1} \mid U\right)^{-1} \circ(f \mid R)^{-1}\right)(Q): R \in \mathcal{F}\right. \text { and } \\
& \left.\qquad Q \in \mathcal{S} \text { with } c^{-1} Q \subset f(R)\right\}
\end{aligned}
$$

and $n_{k+1}(V):=n_{k}(U)+m+2$ for all $V \in \mathcal{F}_{U}$. Again properties (i) and (ii) follow directly. The diameter of any $R \in \mathcal{F}$ ensures that, due to (10), $f \mid R$ is injective and its distortion is close to 1 , say bounded by $K$. From the mean value theorem we can deduce that

$$
\operatorname{diam}(f(R)) \geq \frac{1}{\sqrt{2}} \inf _{z \in R}\left|f^{\prime}(z)\right| \exp \left(-\left|f^{m}(s)\right|^{\varepsilon / 2}\right) \geq \sup _{z \in f(R)}|z|^{1-\delta}
$$

for $M_{0}$ large enough. Note that (19) also yields $|f(z)| \geq \exp \left(\left|f^{m}(s) / 2\right|^{\varepsilon}\right)$ for $z \in R$. By the same arguments as above, (b) and (7) imply that

$$
\begin{aligned}
\frac{\operatorname{meas}\left(f(R) \backslash \bigcup_{Q \in \mathcal{S}, c^{-1} Q \subset f(R)} Q\right)}{f(R)} & \leq 5 B K^{2} \sup _{z \in f(R)}|z|^{\beta+2 \delta-1} \\
& \leq 5 B K^{2} \exp \left((\beta+\delta-1)\left|f^{m}(s) / 2\right|^{\varepsilon}\right),
\end{aligned}
$$

where the last inequality may be deduced from (a) and (19). Since the distortion of $f \mid R$ is bounded by $K$, we can transfer this with the help of (6) to $R$ losing only a factor of $K^{2}$. This estimate for the density in every $R \in \mathcal{F}$ implies the same for their union $\bigcup \mathcal{F}$, which is contained in $f^{m+1}(S)$. More 
precisely, we see that

$$
\begin{aligned}
& \frac{\operatorname{meas}\left(\bigcup \mathcal{F} \backslash \bigcup_{R \in \mathcal{F}, Q \in S, c^{-1} Q \subset f(R)}(f \mid R)^{-1}(Q)\right)}{\operatorname{meas}\left(f^{m+1}(S)\right)} \\
& \quad \leq 5 B K^{4} \exp \left((\beta+\delta-1)\left|f^{m}(s) / 2\right|^{\varepsilon}\right) .
\end{aligned}
$$

From the distortion estimates above, (6), (29) and (30) it follows that

$$
\begin{aligned}
& \frac{\operatorname{meas}\left(U \backslash \bigcup \mathcal{F}_{U}\right)}{\operatorname{meas}(U)} \\
& \leq \frac{\widetilde{K}^{2} K^{4} C^{2 k_{s}} \operatorname{meas}\left(f^{m+1}(S) \backslash \bigcup_{R \in \mathcal{F}, Q \in S, c^{-1} Q \subset f(R)}(f \mid R)^{-1}(Q)\right)}{\operatorname{meas}\left(f^{m+1}(S)\right)} \\
& \leq \frac{\left.\widetilde{K}^{2} K^{4} C^{2 k_{s}} \operatorname{meas}\left(f^{m+1}(S) \backslash \bigcup \mathcal{F}\right)\right)}{\operatorname{meas}\left(f^{m+1}(S)\right)} \\
& +\frac{\widetilde{K}^{2} K^{4} C^{2 k_{s}} \operatorname{meas}\left(\bigcup \mathcal{F} \backslash \bigcup_{R \in \mathcal{F}, Q \in S, c^{-1} Q \subset f(R)}(f \mid R)^{-1}(Q)\right)}{\operatorname{meas}\left(f^{m+1}(S)\right)}
\end{aligned}
$$

$\leq \widetilde{K}^{2} K^{4} C^{2 k_{s}} \exp \left(-\left|f^{m}(s) / 2\right|^{\varepsilon / 2}\right)+5 K^{8} \widetilde{K}^{2} C^{2 k_{s}} \exp \left((\beta+\delta-1)\left|f^{m}(s) / 2\right|^{\varepsilon}\right)$, which, together with (20), is again far stronger than condition (iii) for $\delta$ small and $M_{0}$ large enough.

Setting $\mathcal{F}_{k+1}:=\bigcup_{U \in \mathcal{F}_{k}} \mathcal{F}_{U}$ completes the recursive definition, and all required properties are satisfied. This completes the proof of the theorem.

4. Entire functions. In this section we will only work with functions of the same type as in Theorem 1.2. First of all we will prove some general properties and introduce some notations which will frequently occur. Throughout the section let $P$ and $Q$ be polynomials with $P$ not zero and $Q$ not constant, $c \in \mathbb{C}$ and

$$
f(z):=\int_{0}^{z} P(t) \exp (Q(t)) d t+c .
$$

For $k \in\{1, \ldots, \operatorname{deg}(Q)\}$ define

$$
\phi_{k}:=\frac{(2 k+1) \pi-\arg (q)}{\operatorname{deg}(Q)},
$$

where $q$ denotes the leading coefficient of $Q(z)=q z^{\operatorname{deg}(Q)}+\cdots$. For $R \rightarrow \infty$ the modulus of $\exp (Q(R \exp (\phi i)))$ decreases very fast, and $f(R \exp (\phi i))$ converges to the point

$$
s_{k}:=\lim _{R \rightarrow \infty} \int_{0}^{R \exp (i \phi)} P(t) \exp (Q(t)) d t+c,
$$


which therefore is an asymptotic value of $f$. For $z \in \mathbb{C}$ choose $k$ such that

$$
\phi_{k}-\frac{\pi}{\operatorname{deg}(Q)} \leq \arg (z)<\phi_{k}+\frac{\pi}{\operatorname{deg}(Q)}
$$

and define $\bar{s}(z)=s_{k}$. Let $A$ denote the set of asymptotic values.

LEMMA 4.1.

$f(z)=\bar{s}(z)+\frac{P(z) \exp (Q(z))}{Q^{\prime}(z)}+O\left(|z|^{\operatorname{deg}(P)-\operatorname{deg}(Q)}\right) \exp (Q(z)) \quad$ as $z \rightarrow \infty$.

Proof. Let $z \in \mathbb{C}$. We define $w:=2|z| \exp \left(\phi_{k} i\right)$ with the $k$ as above. Instead of integrating from 0 to $z$ along a segment, one might as well go from 0 to infinity in the direction $\phi_{k}$, come back the same way up to $w$, and finally move forward to $z$. If $z, w$ are not zeros of $Q^{\prime}$, one can find a path from $w$ to $z$ avoiding these zeros such that integration by parts yields

$$
\begin{aligned}
f(z)= & s_{k}+\frac{P(z) \exp (Q(z))}{Q^{\prime}(z)}-\frac{P(w) \exp (Q(w))}{Q^{\prime}(w)} \\
& +\int_{w}^{z}\left(\frac{P^{\prime} Q^{\prime}-P Q^{\prime \prime}}{\left(Q^{\prime}\right)^{2}}\right)(t) \exp (Q(t)) d t \\
& -\int_{2|z|}^{\infty} P\left(t \exp \left(\phi_{k} i\right)\right) \exp \left(Q\left(\exp \left(\phi_{k} i\right)\right)\right) d t .
\end{aligned}
$$

It is easy to obtain estimates of the last three terms that imply the claim.

Lemma 4.2. For every $\delta, \delta^{\prime}>0$ there exists $M>0$ such that for every $z \in G:=\left\{z:|\operatorname{Re}(Q(z))| \geq|z|^{\delta}\right\} \cap D(M)$ the restriction of $f$ to $B\left(z,\left(1-\delta^{\prime}\right) \pi /\left|Q^{\prime}(z)\right|\right)$ is injective.

Proof. We will apply Lemma 2.7. Assume the existence of $z, w \in G$ with $f(z)=f(w)$ and $|z-w|<\left(2-2 \delta^{\prime}\right) \pi /\left|Q^{\prime}(z)\right|$. Then $f([z, w])$ is a closed curve with a singularity of $f^{-1}$ in a bounded component of its complement. The condition $|f(z)-s|=|f(w)-s|$ together with Lemma 4.1 implies

$$
1-\delta^{\prime} / 8<|\operatorname{Re}(Q(w))| /|\operatorname{Re}(Q(z))|<1+\delta^{\prime} / 8
$$

for $|w|$ large enough. Then

$$
1-\delta^{\prime} / 4<|\operatorname{Re}(Q(x))| /|\operatorname{Re}(Q(z))|<1+\delta^{\prime} / 4
$$

for every $x \in[z, w]$. Again by Lemma 4.1 we have

$$
1-\delta^{\prime} / 2<|f(x)| /|f(z)|<1+\delta^{\prime} / 2
$$

for $|z|$ large enough. Therefore the length of the curve $f([z, w])$ must be at least $\pi\left(2-\delta^{\prime}\right)|f(z)|$. On the other hand, the mean value theorem does not 
allow this length to exceed $|z-w| \max _{x \in[z, w]}\left|f^{\prime}(x)\right|$, which is smaller than

$$
\frac{\pi\left(2-\delta^{\prime}-\delta^{\prime 2}\right) \max _{x \in[z, w]}\left|Q^{\prime}(x)\right|}{\left|Q^{\prime}(z)\right|}|f(z)|,
$$

contradicting the above estimate for $|z|$ large enough. Thus the claim follows from Lemma 2.7.

Now we prove the first of the results given in the introduction.

Proof of Theorem 1.2. We verify the properties of Theorem 3.1. Since every $s \in A$ escapes exponentially, there exists $\delta_{s}>0$ such that $\left|f^{n+1}(s)\right| \geq$ $\exp \left(\left|f^{n}(s)\right|^{\delta_{s}}\right)$ for almost every $n \in \mathbb{N}$. Suppose $0<\delta<\min _{s \in A} \delta_{s}$. By Lemma 4.1 we have an estimate for $|f|$. For $\varepsilon<\delta$ and $\delta_{1}<\operatorname{deg}(Q)-1$ $<\delta_{2}$ property (a) follows if we redefine $\bar{s}$ as zero on the part of $G$ where $\operatorname{Re}(Q(z))>0$.

Far away from the origin, $\mathbb{C} \backslash G$ consists of neighborhoods around the pre-images under $Q$ of the imaginary axis, whose widths at a distance $R$ from the origin are of magnitude $R^{-\operatorname{deg}(Q)+1+\delta}$. By the width at distance $R$ from the origin we mean the diameter of the largest disc that is contained in the set and whose center has modulus $R$. For $-\operatorname{deg}(Q)+1+\delta<\beta<1$ and $B$ sufficiently large, (b) follows.

As mentioned above, we have $\left|f^{n+1}(s)\right| \geq \exp \left(\left|f^{n}(s)\right|^{\delta_{s}}\right)$ except for a finite number of $n \in \mathbb{N}$. Thus the real part of $Q\left(f^{n}(s)\right)$ is at least of magnitude $\left|Q\left(f^{n}(s)\right)\right|^{\delta_{s} / \operatorname{deg}(Q)}$. The distance of $f^{n}(s)$ to $\mathbb{C} \backslash G$ is therefore no less than $\left|f^{n}(s)\right|^{-\operatorname{deg}(Q)+1+\delta_{s}}$. If we choose $\tau$ such that $\beta<\tau<$ $-\operatorname{deg}(Q)+1+\min _{s \in A} \delta_{s}$, then (c) is satisfied. Now we can apply Theorem 3.1 and get meas $(T(f))>0$. Case (ii) of Theorem 1.1 follows. As explained in Remark 3.2 we know that $T(f) \subset J(f)$ due to a result of I. N. Baker [1].

Assume now $\operatorname{deg}(Q) \geq 3$. Then $\operatorname{deg}(Q)-1-\delta>1$ for $\delta$ small enough. This implies that meas $(\mathbb{C} \backslash G)<\infty$ and if $\delta_{1}>1$ even that the measure of $\left\{z: \operatorname{dist}(z, \mathbb{C} \backslash G) \leq|z|^{-\delta_{1}}\right\}$ is finite. This follows since this set is contained in

$$
B(0, M) \cup \bigcup_{k=1}^{\operatorname{deg}(Q)}\left\{z:\left|\arg (z)-\frac{(4 k+1) \pi-2 \arg (q)}{2 \operatorname{deg}(Q)}\right| \leq 2 q|z|^{\delta-\operatorname{deg}(Q)}\right\} .
$$

Using polar coordinates one sees that the measure of the last set is bounded by

$$
\operatorname{deg}(Q) \int_{M}^{\infty} R^{1-\operatorname{deg}(Q)+\delta} d 2 q R=\frac{2 q \operatorname{deg}(Q) M^{2-\operatorname{deg}(Q)+\delta}}{2-\operatorname{deg}(Q)+\delta} .
$$

We cover the set $\left\{z: \operatorname{dist}(z, \mathbb{C} \backslash G) \geq 2|z|^{-\delta_{1}}\right\}$ with a family $\mathcal{S}$ of squares $S \subset\left\{z: \operatorname{dist}(z, \mathbb{C} \backslash G) \geq|z|^{-\delta_{1}}\right\}$ whose diameters satisfy $\sup _{z \in S}|z|^{-\delta_{2}} \leq$ $\operatorname{diam}(S) \leq 4 \sup _{z \in S}|z|^{-\delta_{2}}$. The density of $\mathcal{F}(f)$ in any $S \in \mathcal{S}$ is, due to Theorem 3.1, at most $\exp \left(-\eta \inf _{z \in S}|z|^{\varepsilon}\right)$. Let $R_{k}:=M+k$ for all $k \in \mathbb{N} \cup\{0\}$. 
Then it follows that

$$
\begin{aligned}
& \operatorname{meas}(F(f)) \leq \operatorname{meas}\left(\left\{z: \operatorname{dist}(z, \mathbb{C} \backslash G) \leq 2|z|^{-\delta_{1}}\right\}\right)+\sum_{S \in \mathcal{S}} \operatorname{meas}(\mathcal{F}(f) \cap S) \\
& \leq \pi M^{2}+\frac{2 q \operatorname{deg}(Q) M^{2-\operatorname{deg}(Q)+\delta}}{2-\operatorname{deg}(Q)+\delta}+\sum_{k \in \mathbb{N}} \sum_{R_{k}<\mathcal{S}} \operatorname{meas}(\mathcal{F}(f) \cap S) \\
& \leq \pi M^{2}+\frac{2 q \operatorname{deg}(Q) M^{2-\operatorname{deg}(Q)+\delta}}{2-\operatorname{deg}(Q)+\delta}+\sum_{k=1}^{\infty} 2 \pi\left(R_{k}+1\right) \exp \left(-\eta R_{k+1}^{\varepsilon}\right)
\end{aligned}
$$

which is finite. This gives the second part of Theorem 1.2.

This allows us to construct examples of functions $f$ for which we have $0<\operatorname{meas}(\mathcal{F}(f))<\infty$. For example we can choose the parameters $a, b$ (e.g. $\left.a=\left(27 \pi^{2} / 16\right)^{1 / 3}, b=\log (\sqrt{a / 3})\right)$ so that both critical points of the function

$$
f(z)=\exp \left(z^{3}+a z+b\right)
$$

are fixed, and the only asymptotic value 0 escapes to infinity on the real axis. Thus the Fatou set of $f$, which consists of those two super-attractive basins, has finite measure. In Figure 3, where the part $\{z:|\operatorname{Re}(z)| \leq 2,|\operatorname{Im}(z)| \leq 2\}$ of the plane is displayed, these two super-attractive basins are coloured black. We should note that the existence of such examples is not very surprising after the construction of examples with a positive measure Julia set by C. McMullen in [18]. Also the idea of using concrete measure es-

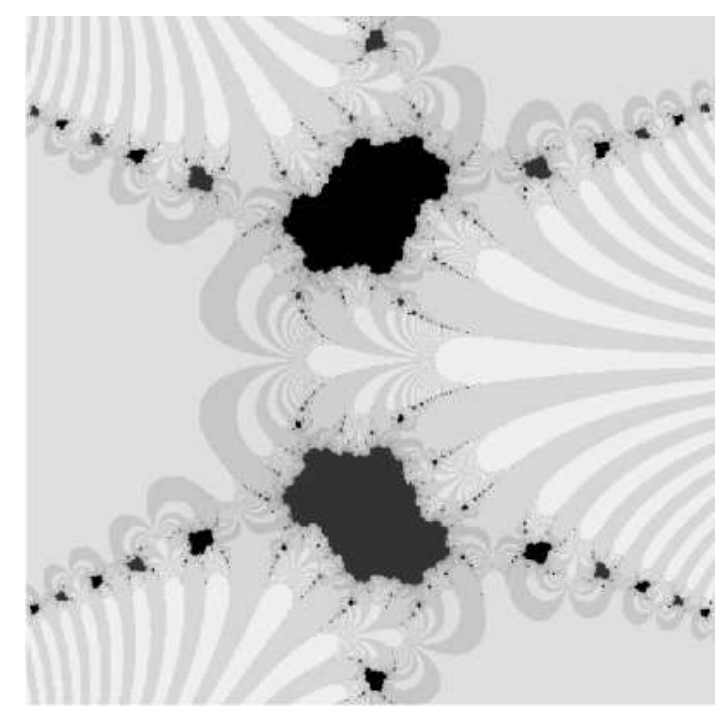

Fig. 3. The Fatou set of $f(z)=\exp \left(z^{3}+a z+b\right)$ with $a=\left(27 \pi^{2} / 16\right)^{1 / 3}$ and $b=\log (\sqrt{a / 3})$ 
timates like the one in Theorem 3.1 in order to show finite measure of subsets of the Fatou set has been used before by H. Schubert, who proved in [26] that the measure of the Fatou set of the sine function in the strip $\{z: 0 \leq \operatorname{Re}(z) \leq 2 \pi\}$ is finite, as conjectured by J. Milnor in [20].

We choose $0<\delta<\min _{a, b \in A} \operatorname{dist}(a, b) / 2, R>\max _{a \in A}|a|$ and assume that $M$ is sufficiently large. Then for any $z \in G$, either there will be exactly one $a \in A$ for which $|f(z)-a|<\delta$, or $|f(z)| \geq R$. For $a \in A$ we denote by $G_{a}$ and $G_{\infty}$ the parts of $G$ for which the respective conditions are satisfied. In order to prove Theorem 1.3 we need the following lemma.

Lemma 4.3. Let $\Gamma:=\bigcup_{n \in \mathbb{N}}\left(f^{n}\right)^{-1}(B(a, \varepsilon))$ for some $a \in A$ and $\varepsilon>0$. Then there exist positive constants $c, C$, and a family $\mathcal{F}$ of disjoint domains $D$ such that

$\operatorname{diam}(D) \leq \frac{C}{\sup _{z \in D}\left|Q^{\prime}(z)\right|}, \quad \frac{\operatorname{meas}(\Gamma \cap D)}{\operatorname{meas}(D)} \geq c, \quad$ and $\quad \operatorname{meas}\left(G_{s} \backslash \bigcup \mathcal{F}\right)=0$ if $s=\infty$ or if $s$ is an asymptotic value that escapes exponentially.

Proof. Since $a$ is an asymptotic value, we have $\lim _{R \rightarrow \infty} f\left(R \exp \left(\phi_{a} i\right)\right)=a$ for some $k \in\{0,1, \ldots, \operatorname{deg} Q\}$ and

$$
\phi_{a}=\frac{(2 k+1) \pi-q}{\operatorname{deg} Q} .
$$

From Lemma 4.1 it follows that for any $\delta^{\prime}>0$ there exists $M>0$ such that $f^{-1}(B(s, \varepsilon))$ contains the set

$$
\left\{z: \phi_{a}-\frac{\left(1-\delta^{\prime}\right) \pi}{2 \operatorname{deg}(Q)}<\arg (z)<\phi_{a}+\frac{\left(1-\delta^{\prime}\right) \pi}{2 \operatorname{deg}(Q)}\right\} \cap D(M) .
$$

Since Lemma 4.1 also gives good estimates for the argument of $f$ in $G_{\infty}$ it follows that the set

$$
\left\{z: \phi_{a}-\frac{\left(1-2 \delta^{\prime}\right) \pi}{2 \operatorname{deg}(Q)}<\arg \left(\frac{P(z)}{Q^{\prime}(z)}\right)+\operatorname{Im}(Q(z))<\phi_{a}+\frac{\left(1-2 \delta^{\prime}\right) \pi}{2 \operatorname{deg}(Q)}\right\} \cap G_{\infty}
$$

is contained in $f^{-2}(B(s, \varepsilon))$. Every component of this set is an unbounded region whose width at distance $R$ from the origin is at least

$$
\frac{1-3 \delta^{\prime}}{\operatorname{deg}(Q)} 2 \pi|q|^{-1} R^{1-\operatorname{deg}(Q)}
$$

for sufficiently large $R$. We refer to these regions as "channels"; see the left side of Figure 4, where these channels are coloured black. In order to be able to display the structure we had to magnify their diameter relative to $M$. The "gaps" in between these channels have a width of at most $\left(2-\left(1-3 \delta^{\prime}\right) / \operatorname{deg}(Q)\right) \pi|q|^{-1} R^{1-\operatorname{deg}(Q)}$, still assuming that $M$ is large. The complement of $\Gamma$ in $G_{\infty}$ must lie in the gaps between these channels. For $\delta^{\prime}$ sufficiently small, simple geometric arguments give for any constant $C^{\prime}>\sqrt{2} \pi(2-1 / \operatorname{deg}(Q))$ a constant $c^{\prime}>0$ such that for any square $S$ 

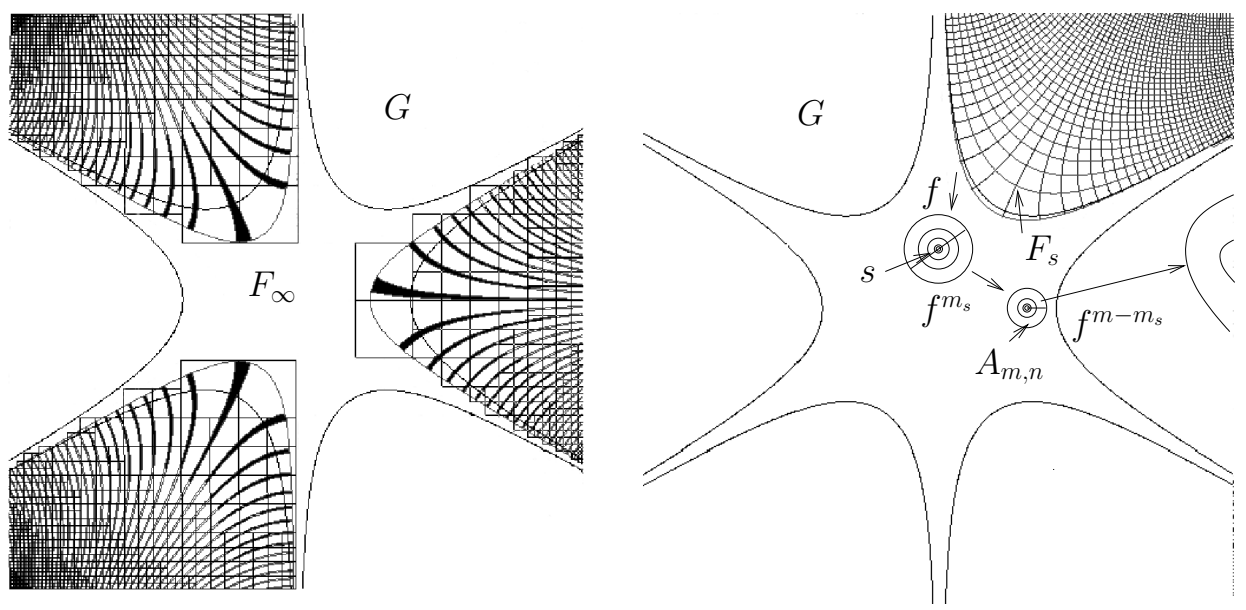

Fig. 4. Construction of the family $\mathcal{F}$

intersecting $G_{\infty}$ and satisfying $\operatorname{diam}(S) \geq C^{\prime}\left(\inf _{z \in S}|Q(z)|\right)^{-1}$, the density of $\Gamma$ in $S$ is bounded below by $c^{\prime}$. (For $C^{\prime}$ sufficiently large one can choose $c^{\prime}$ arbitrarily close to $\left.1 / 2 \operatorname{deg}(Q)\right)$.

We cover $G_{\infty}$ up to a set of measure zero by a family $\mathcal{F}_{\infty}$ of squares $S$ with $C^{\prime}\left(\inf _{z \in S}\left|Q^{\prime}(z)\right|\right)^{-1}<\operatorname{diam}(S)<4 C^{\prime}\left(\sup _{z \in S}\left|Q^{\prime}(z)\right|\right)^{-1}$. We obtain this family in a similar way to the family $\mathcal{S}$ in the proof of Theorem 3.1. We begin with a grid of open squares with a constant diameter, covering the whole plane, subdivide each into four parts until they satisfy the upper bound, and finally discard those not intersecting $G_{\infty}$. Then our conditions are satisfied with $c=c^{\prime}$ and $C=4 C^{\prime}$. The family $\mathcal{F}_{\infty}$ could look similar to the left side of Figure 4.

Now we need to find a covering $\mathcal{F}_{s}$ of $G_{s}$ for every asymptotic value $s$ that escapes exponentially. We define $m_{s} \in \mathbb{N} \cup\{0\}$ as before: minimal such that for $m \geq m_{s}$ the point $f^{m}(s)$ is not a critical point of $f$, and choose $m_{0} \geq m_{s}$ such that for $m \geq m_{0}, f^{m}(s)$ is not a critical point of $Q$. Then we choose $n_{m}$ to be the smallest natural number for which there exists $l_{m} \leq 4$ with

$$
\left|\frac{\left(f^{m-m_{s}}\right)^{\prime}\left(f^{m_{s}}(s)\right) Q^{\prime}\left(f^{m}(s)\right)}{\left(f^{m-m_{s}-1}\right)^{\prime}\left(f^{m_{s}}(s)\right) Q^{\prime}\left(f^{m-1}(s)\right)}\right|=l_{m}^{n_{m}} .
$$

The exponential escape of $s$ implies that $l_{m}^{n_{m}}$ is of magnitude $\left|f^{m}(s)\right|$, so that $l_{m} \rightarrow 4$ and $n_{m} \rightarrow \infty$ as $m \rightarrow \infty$. For $0 \leq n \leq n_{m}$ we define

$$
R_{m, n}:=\left|\frac{\left(1-\delta^{\prime}\right) l_{m}^{n} \pi}{2\left(f^{m-m_{s}}\right)^{\prime}\left(f^{m_{s}}(s)\right) Q^{\prime}\left(f^{m}(s)\right)}\right|
$$

and for $n \neq 0$ we consider the slit annulus

$$
A_{m, n}:=\left\{z: R_{m, n-1}<\left|z-f^{m_{s}}(s)\right|<R_{m, n}, z-f^{m_{s}}(s) \notin \mathbb{R}_{>0}\right\} .
$$


Let $\mathcal{F}_{s}$ be the family of all connected components of $\left(f^{m_{s}+1}\right)^{-1}\left(A_{m, n}\right)$ intersecting $G_{s}$, for $m \geq m_{0}$ and $n \in\left\{1, \ldots, n_{m}\right\}$. We try to give an idea of $\mathcal{F}_{s}$ in Figure 4. For $M$ large enough these components cover $G_{s}$ up to a set of measure zero. Next we will verify the diameter condition. For large $m$ the annulus $A_{m, k}$ is very close to $f^{m_{s}}(s)$, so that the power series of $f^{m_{s}}$ gives good estimates. If $k_{s}$ is the multiplicity of $f^{m_{s}}$ in $s$, there are $k_{s}$ pre-images $A^{\prime}$ of $A_{m, k}$ under $f^{m_{s}}$ which are contained in $B(s, r) \backslash B\left(s, r^{-}\right)$with

$$
r^{(-)}:=\frac{1+(-) \delta^{\prime}}{\left|\left(f^{m_{s}}\right)^{\left(k_{s}\right)}(s)\right|}\left|R_{m, n(-1)}\right|^{1 / k_{s}} .
$$

Since the ratio of the outer and inner radii of this annulus is $\frac{1+\delta^{\prime}}{1-\delta^{\prime}} l_{m}^{1 / k_{s}}$, the distortion of $f^{m_{s}}$ on these $A^{\prime}$ is bounded by any constant $C_{2}$ which is larger than this ratio to the power of $k_{s}-1$ for $m_{0}$ large enough.

Every connected component $D$ of $f^{-1}\left(A^{\prime}\right)$ intersecting $G_{s}$, which is therefore an element of $\mathcal{F}_{s}$, is a simply connected domain with a diameter of at most $C \inf _{z \in D}\left|Q^{\prime}(z)\right|^{-1}$ for any $C>4^{2 / k_{s}}+24^{1 / k_{s}} \pi / k_{s}$ and $M$ large enough. This follows since we can connect any two points in $A^{\prime}$ by a path in $A^{\prime}$ whose length is at most $\left(2 \pi / k_{s}+l_{m}^{1 / k_{s}}\right) r$, and for $z \in D$ we know from Lemma 4.1 that $\left|f^{\prime}(z)\right|$ is bounded by $\left|Q^{\prime}(z)\right| r^{-}$. Thus the diameter condition in the claim is satisfied. An analogous upper estimate for $\left|f^{\prime}\right|$ on $D$ implies that the distortion of $f$ on such a domain $D$ is bounded by any constant $C_{3}>4^{1 / k_{s}}$ if $M$ is large enough.

It remains to show that the density of $\Gamma$ in these pre-images is again bounded away from zero by some $c>0$.

The diameter of $A_{m, n_{m}}$ is chosen in such a way that for $m$ large enough $f^{m-m_{s}}$ is injective on this set and its distortion is bounded by $K_{\widetilde{c}}$ from Lemma 2.4 with $\widetilde{c}<1 / \sqrt{2} l_{m}^{n_{m}-n}$. This follows since for $m$ large enough, $f^{m-m_{s}-1}$ is injective on $B\left(f^{m_{s}}, R_{m-2,0}\right)$ so that its distortion on $A_{m, n}$ is bounded by any constant larger than 1 , say $\left(1-\delta^{\prime} / 2\right) /\left(1-\delta^{\prime}\right)$. Thus we have

$$
f^{m-m_{s}-1}\left(A_{m, n}\right) \subset B\left(f^{m-1}(s), \frac{\left(1-\delta^{\prime} / 2\right) \pi}{2\left|Q^{\prime}\left(f^{m-1}(s)\right)\right|}\right) .
$$

By Lemma 4.2 we know that $f$ is injective on the ball with the same center but twice the radius, and the distortion estimate for $f^{m-m_{s}} \mid A_{m, n}$ follows from Lemma 2.4.

Since $s$ escapes exponentially we may assume that $\delta$ is small enough to ensure $\left|f^{n+1}(s)\right| \geq \exp \left(\left|f^{n}(s)\right|^{2 \delta}\right)$ for large $n$, and in particular $f^{n}(s) \in G_{\infty}$.

To show that the density of $\Gamma$ in $f^{m-m_{s}}\left(A_{m, n}\right)$ is bounded below by some $c>0$, we distinguish between large and small $n$.

If $n$ is so small that $l_{m}^{n} \leq\left|f^{m}(s)\right|^{\delta}$, it follows from the definition of $n_{m}$ that $n_{m}-n$ is large. Assuming $\delta<1 / 2$ we get $n_{m}-n \geq n_{m} / 2$ for $m$ large 
enough. Thus the distortion of $f^{m-m_{s}}$ on $A_{m, n}$ is bounded by some $K_{m}$, which tends to 1 as $m \rightarrow \infty$. Thus for $m$ large the set $f^{m-m_{s}}\left(A_{m, n}\right)$ is close to the annulus

$$
B\left(f^{m}(s),\left|\frac{\left(1-\delta^{\prime}\right) l_{m}^{n} \pi}{2 Q^{\prime}\left(f^{m}(s)\right)}\right|\right) \backslash B\left(f^{m}(s),\left|\frac{\left(1-\delta^{\prime}\right) l_{m}^{n-1} \pi}{2 Q^{\prime}\left(f^{m}(s)\right)}\right|\right)
$$

in the sense that as $m \rightarrow \infty$ the density of the complement of $f^{m-m_{s}}\left(A_{m, n}\right)$ in this annulus tends to zero. This annulus is contained in $G_{\infty}$ and its diameter is more than twice the width of the gaps in between the channels of $\Gamma$. Thus it has to intersect these channels. More precisely, the diameter ensures that the density of $\Gamma$ in $f^{m-m_{s}}\left(A_{m, n}\right)$ is bounded below by a positive constant $c_{2}$. Since the distortion of $f^{m-m_{s}}$ on $A_{m, n}$ is arbitrarily close to 1 for large $m$, for $c_{3}<c_{2}$ this carries over by (6) to

$$
\frac{\operatorname{meas}\left(A_{m, n} \cap \Gamma\right)}{\operatorname{meas}\left(A_{m, n}\right)}>c_{3} \text {. }
$$

For larger $n$ the distortion is still bounded by $K:=K_{1 / \sqrt{2}}$, so that one could call $f^{m-m_{s}}\left(A_{m, n}\right)$ a $K$-quasi-annulus, whose center $f^{m}(s)$ lies in $G_{\infty}$ and whose diameter is far larger than the gaps in between the channels of $\Gamma$. We choose

$$
0<c_{4}<\sin \left(\frac{\pi}{2 \operatorname{deg}(Q)}\right) / K
$$

and $z \in \partial B\left(f^{m_{s}}(s),\left(R_{m, n}+R_{m, n-1}\right) / 2\right)$ maximizing the distance of $f^{m-m_{s}}(z)$ to $\mathbb{C} \backslash G_{\infty}$. For large $m$ it follows that $f^{m-m_{s}}\left(B\left(z, c_{4} R_{m, n}\right)\right) \subset G_{\infty}$, since the choice of $c_{4}$ guarantees that $f^{m-m_{s}}\left(B\left(z, c_{4} R_{m, n}\right)\right)$ lies in a sector of angle $\pi / \operatorname{deg}(Q)$ and vertex $f^{m}(s)$, and the boundary of the component of $G_{\infty}$ containing $f^{m}(s)$ is tangent to the boundary of a sector of the same angle and vertex 0 . This is displayed in Figure 5 . We note that $c_{4}<1 / 4$,

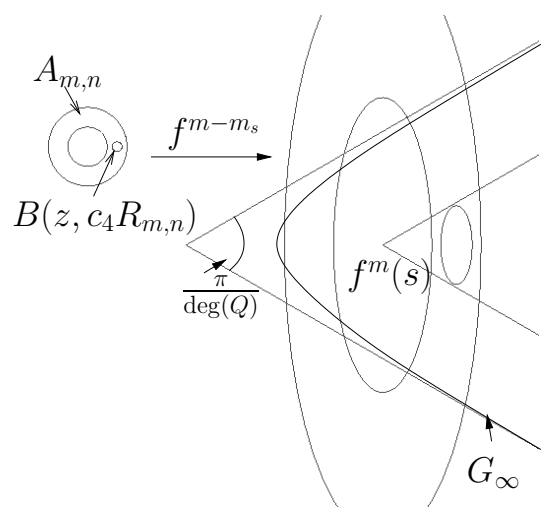

Fig. 5. Constructions to estimate the density of $\Gamma$ in $A_{m, n}$ 
so that $B\left(z, c_{4} R_{m, n}\right) \subset A_{m, n}$. Using the family $\mathcal{F}_{\infty}$ defined above as a cover, one sees that the density of $\Gamma$ in $f^{m-m_{s}}\left(B\left(z, c_{4} R_{m, n}\right)\right)$ is bounded below by any $0<c_{5}<c^{\prime}$ if $m$ large enough. This carries over by (6) to $B\left(z, c_{4} \operatorname{diam}\left(f^{m-m_{s}}\left(A_{m, n}\right)\right)\right)$, in which the density of $\Gamma$ is at least $c_{5} / K^{2}$. Assuming $c_{3} \leq c_{5} c_{4}^{2} / K^{2}$ we get (33) for all $n$ and $m$ if $m_{0}$ is large enough. By (6) this carries over to the elements of $\mathcal{F}_{s}$ and completes the proof for $c=c_{3} / C_{2}^{2} C_{3}^{2}$.

Lemma 4.4. Let $B \subset \mathbb{C}$ be finite and such that every $b \in B$ escapes exponentially. Suppose that every singularity of the inverse is either pre-periodic, escapes exponentially, or is contained in some attractive basin. Suppose further that $A \not \subset O^{+}(B)$. Then the set $\left\{z \in J(f): \omega(z) \subset O^{+}(B) \cup\{\infty\}\right\}$ has zero measure.

Proof. We assume that $\widetilde{X}:=\left\{z \in J(f): \omega(z) \subset O^{+}(B) \cup\{\infty\}\right\}$ has positive measure. Then we find $\varepsilon>0$ such that

$$
X:=\left\{z \in \tilde{X}: \omega(z) \neq\{\infty\} \text { and } \operatorname{dist}\left(O^{+}(z), a\right)>\varepsilon, \forall a \in A \backslash O^{+}(B)\right\}
$$

also has positive measure. We may assume $\omega(z) \neq\{\infty\}$ since A. Eremenko and M. Lyubich [10] proved that the set $I(f)$ of escaping points has measure zero. The assumption $\operatorname{dist}\left(O^{+}(z), a\right)>\varepsilon$ is permissible since $X$ is contained in the countable union of the sets $\left\{z: \operatorname{dist}\left(a, O^{+}(z)\right)>\varepsilon_{n}>0, \forall a \in A\right\}$ where $\varepsilon_{n} \rightarrow 0$.

Due to the exponential escape of all elements of $B$, we may choose $\delta>0$ such that $\left|f^{n+1}(b)\right| \geq \exp \left(\left|f^{n}(b)\right|^{4 \delta}\right)$ for every $b \in B$ and $n$ large enough. Let $z_{0}$ be a density point of $X$. Since the iterates of $z_{0}$ do not tend to infinity, there exists a convergent subsequence $f^{\beta(n)}\left(z_{0}\right)$, whose limit must be of the form $f^{n_{0}}(b)$ with $n_{0} \in \mathbb{N} \cup\{0\}$ and $b \in B$. We may assume $n_{0} \geq m_{b}:=\max \left(\left\{m \in \mathbb{N}: f^{\prime}\left(f^{m-1}(b)\right)=0\right\} \cup\{0\}\right)$. For all $n \in \mathbb{N}$ we take $\alpha(n) \geq \beta(n)$ smallest possible with

$$
\left|f^{\alpha(n)}\left(z_{0}\right)-f^{\alpha(n)-\beta(n)+n_{0}}(b)\right| \geq\left|f^{\alpha(n)}\left(z_{0}\right)\right|^{1-\operatorname{deg}(Q)+3 \delta}
$$

and $B_{n}:=B\left(f^{\alpha(n)}\left(z_{0}\right),\left|f^{\alpha(n)}\left(z_{0}\right)\right|^{1-\operatorname{deg}(Q)+2 \delta}\right)$. We will see that for large $n$ the inverse branch $g_{n}$ of $f^{\alpha(n)}$ mapping $f^{\alpha(n)}\left(z_{0}\right)$ to $z_{0}$ may be extended to $B_{n}$ with uniformly bounded distortion. Furthermore we show that the density of $X$ in $B_{n}$ does not tend to 1 . This carries over to $g_{n}\left(B_{n}\right)$ by (6). Finally, we show $\operatorname{diam}\left(g_{n}\left(B_{n}\right)\right) \rightarrow 0$, a contradiction to the choice of $z_{0}$ as a density point of $X$.

Since $\operatorname{sing}\left(f^{-1}\right)$ is bounded, we can extend every branch of $f^{-1}$ to every slit annulus around the origin whose inner radius is larger than the modulus of every singularity of the inverse. For large $n$ the diameter of $B_{n}$ and the definition of $\alpha$ ensure that we can extend the branch of $f^{-1}$ mapping $f^{\alpha(n)}\left(z_{0}\right)$ to $f^{\alpha(n)-1}\left(z_{0}\right)$ to $B_{n}$ so that the distortion is bounded by a constant, which can be chosen arbitrarily close to 1 , and for the image $B_{n}^{-1}$ it 
follows that

$$
\frac{\operatorname{diam}\left(B_{n}^{-1}\right)}{\left|f^{\alpha(n)-1}\left(z_{0}\right)-f^{n_{0}+\alpha(n)-\beta(n)-1}(b)\right|} \leq\left|f^{\alpha(n)-1}\left(z_{0}\right)\right|^{-\delta / 2}
$$

and

$$
B_{n}^{-1} \subset B\left(f^{n_{0}+\alpha(n)-\beta(n)-1}(b), 2\left|f^{n_{0}+\alpha(n)-\beta(n)-1}(b)\right|^{1-\operatorname{deg}(Q)+3 \delta}\right) .
$$

The choice of $n_{0} \geq m_{b}$ ensures that we can apply the same argument as above $\alpha(n)-\beta(n)-1$ times. Thus we can extend the branch of $\left(f^{\alpha(n)-\beta(n)-1}\right)^{-1}$, mapping $f^{n_{0}+\alpha(n)-\beta(n)-1}(b)$ to $f^{n_{0}}(b)$, to a ball around $f^{n_{0}+\alpha(n)-\beta(n)-1}(b)$ with a diameter of almost the modulus of its center. Again this implies that its distortion on $B\left(f^{n_{0}+\alpha(n)-\beta(n)-1}(b), 2\left|f^{n_{0}+\alpha(n)-\beta(n)-1}(b)\right|^{1-\operatorname{deg}(Q)+3 \delta}\right)$ tends to 1 as $n$ tends to infinity. Moreover from (34) it follows that $B_{n}^{-1}$ is mapped to a small ball $B\left(f^{\beta(n)}\left(z_{0}\right), r_{n}\right)$ with $r_{n} /\left|f^{\beta(n)}\left(z_{0}\right)-f^{n_{0}}(b)\right| \rightarrow 0$ as $n \rightarrow \infty$. Since $P(f)$ does not accumulate at $f^{n_{0}}(b)$, we can extend the branch of the inverse of $f^{\beta(n)}$ mapping $f^{\beta(n)}\left(z_{0}\right)$ to $z_{0}$ to $B\left(f^{\beta(n)}\left(z_{0}\right)\right.$, $\left.\left|f^{\beta(n)}\left(z_{0}\right)-f^{n_{0}}(b)\right|\right)$. Thus $g_{n}$ exists and its distortion tends to one as $n$ tends to infinity.

For $s \in A \cup\{\infty\}$ we define $G_{s}$ as in Lemma 4.3. Due to our choice of $\operatorname{diam}\left(B_{n}\right)$, the density of $\mathbb{C} \backslash G$ in $B_{n}$ tends to zero as $n$ tends to infinity. For $M$ large enough and $s \in A \backslash O^{+}(B)$ we have $G_{s} \cap X=\emptyset$. On the other hand, for $s \in A \cap O^{+}(B) \cup\{\infty\}$ we can apply Lemma 4.3 to $\Gamma:=\mathbb{C} \backslash X$ and obtain a family $\mathcal{F}$ of disjoint domains covering $B_{n} \cap G_{s}$ up to a set of measure zero, such that the density of $\Gamma$ in all of these is bounded below by some positive constant $c$. The diameter of these domains is much smaller than the diameter of $B_{n}$, so that we can neglect the ones intersecting the boundary of $B_{n}$. Thus the density of $X$ in $B_{n}$ does not tend to 1 .

It remains to show that $\operatorname{diam}\left(g_{n}\left(B_{n}\right)\right) \rightarrow 0$, which is equivalent to $\left|\left(f^{\alpha(n)}\right)^{\prime}\left(z_{0}\right)\right|\left|f^{\alpha(n)}\left(z_{0}\right)\right|^{\operatorname{deg}(Q)-1-2 \delta} \rightarrow \infty$.

Lemma 4.1 implies the existence of $R>0$ such that

$$
\frac{1}{2}\left|f^{\prime}(z)\right| \leq \frac{|f(z)-\bar{s}(z)|}{\left|Q^{\prime}(z)\right|} \leq 2\left|f^{\prime}(z)\right| \quad \text { for }|z| \geq R .
$$

We choose $\varepsilon^{\prime}>0$ small enough such that for $b \in O^{+}(B) \cap B(0, R)$ and $z \in B\left(s, \varepsilon^{\prime}\right)$ and

$$
m:=\min \left\{m \in \mathbb{N}:\left|f^{m}(z)-f^{m}(s)\right| \geq\left|f^{m}(s)\right|^{1-\operatorname{deg}(Q)+3 \delta}\right\},
$$

we have

$$
\left|\left(f^{m}\right)^{\prime}(z)\right| \geq \frac{2\left|f^{m}(z)\right|^{1-\operatorname{deg}(Q)+9 \delta / 4}}{|z-s|} .
$$

If the distortion of $f^{m}$ on $B(s,|z-s|)$ were bounded by a constant, a stronger statement would follow from the mean value theorem. There are however two obstructions to uniformly bounded distortion. There may 
be critical points on the orbit of $s$ and the distortion of the last iterate $f \mid f^{m-1}(B(s,|z-s|))$ is not necessarily uniformly bounded. Thus in order to obtain the existence of such an $\varepsilon^{\prime}$ we need to consider these iterates separately. For $\varepsilon^{\prime}$ small enough the power series of $f^{m_{s}}$ guarantees that the modulus of the derivative of $f^{m_{s}}$ on $B(s,|z-s|)$ is bounded by $2\left|\left(f^{m_{s}}\right)^{\prime}(z)\right|$. Here $m_{s}$ is again chosen such that $f^{n}(s)$ is not a critical point of $f$ for $n \geq m_{s}$. The choice of $m$ guarantees that the distortion of $f^{m-m_{s}-1} \mid f^{m_{s}}(B(s,|z-s|))$ is arbitrarily close to 1 for $\varepsilon^{\prime}$ small enough and

$$
f^{m-1}(B(s,|z-s|)) \subset B\left(f^{m-1}(s), 2\left|f^{m-1}(s)\right|^{1-\operatorname{deg}(Q)+3 \delta}\right) .
$$

The definition of $\delta$ and the diameter of the latter set ensure that the magnitude of $\operatorname{Re}\left(Q\left(f^{m-1}(x)\right)\right)$ is the same for all $x \in B(s,|z-s|)$. By Lemma 4.1 this carries over to $\left|f^{m}(x)\right|$ and $\left|f^{\prime}\left(f^{m-1}(x)\right)\right|$ and gives estimate (36).

As $\omega\left(z_{0}\right) \subset \overline{O^{+}(B)}$, there exists $j_{0} \in \mathbb{N}$ such that for all $j \geq j_{0}$ the point $f^{j}\left(z_{0}\right)$ is not contained in the compact set $\overline{B(0, R)} \backslash \bigcup_{n \in \mathbb{N}, s \in B} B\left(f^{n}(s), \varepsilon^{\prime}\right)$. Now let $I$ be the set of $j_{0} \leq j \in \mathbb{N}$ such that $f^{j}\left(z_{0}\right) \notin B(0, R)$ and $f^{j+1}\left(z_{0}\right) \in$ $B(0, R)$. Then for every $j_{0} \leq j \notin I$ with $\left|f^{j}\left(z_{0}\right)\right| \geq R$ we have $\left|f^{\prime}\left(f^{j}\left(z_{0}\right)\right)\right| \geq 1$. This follows since otherwise $\left|f^{j+1}(z)-s\right| \leq 2 /\left|Q^{\prime}\left(f^{j}\left(z_{0}\right)\right)\right|$ would imply $j \in I$, if we assume $R$ to be larger than the modulus of every $s \in A$.

Now for $j \in I$ we have

$$
\left|f^{j+1}\left(z_{0}\right)-s\right| \leq \frac{2\left|f^{\prime}\left(f^{j}(s)\right)\right|}{\left|Q^{\prime}\left(f^{j}(s)\right)\right|},
$$

which is at most $\varepsilon^{\prime}$. We assume $\varepsilon^{\prime}<\varepsilon$, so that $s \in O^{+}(B)$. We choose $m_{j}$ as in (35) for $z=f^{j+1}(s)$. Then we have $m_{j} \leq \alpha(n)$, and (36) together with (37) imply that

$$
\begin{aligned}
\left|\left(f^{m_{j}}\right)^{\prime}\left(f^{j}\left(z_{0}\right)\right)\right| & =\left|f^{\prime}\left(f^{j}\left(z_{0}\right)\right)\right|\left|\left(f^{m_{j}-1}\right)^{\prime}\left(f^{j+1}\left(z_{0}\right)\right)\right| \\
& \geq\left|Q^{\prime}\left(f^{j}\left(z_{0}\right)\right)\right|\left|f^{m_{j}+j}\left(z_{0}\right)\right|^{1-\operatorname{deg}(Q)+9 \delta / 4} .
\end{aligned}
$$

Let $\Delta_{j}:=\min \{i \in I \cup\{\alpha(n)\}: i>j\}-m_{j}-j$. We may assume $j_{0} \in I$ since otherwise we continue with $j_{0} \leq j_{0}^{\prime} \in I$ smallest possible. By the chain rule we get

$$
\begin{aligned}
& \frac{\left|\left(f^{\alpha(n)}\right)^{\prime}\left(z_{0}\right)\right|}{\left|f^{\alpha(n)}\left(z_{0}\right)\right|^{1-\operatorname{deg}(Q)+2 \delta}} \\
& =\frac{\left|\left(f^{j_{0}}\right)^{\prime}\left(z_{0}\right)\right|}{\left|f^{\alpha(n)}\left(z_{0}\right)\right|^{1-\operatorname{deg}(Q)+2 \delta}} \prod_{j \in I, j_{0} \leq j<\alpha(n)}\left|\left(f^{\Delta_{j}}\right)^{\prime}\left(f^{m_{j}+j}\left(z_{0}\right)\right)\right|\left|\left(f^{m_{j}}\right)^{\prime}\left(f^{j}\left(z_{0}\right)\right)\right| .
\end{aligned}
$$

From (38) we obtain a lower estimate of the product above, in which most of the factors in the product cancel out each other. More precisely, for each $j \in I$ except the first and the last in the product, the factor $\left|f^{m_{j}+j}\left(z_{0}\right)\right|$ only remains with a power of $9 / 4$. If $\Delta_{j}=0$ this follows directly from (38) 
by considering the $j$ th and the $(j+1)$ st factor of the product together. Of course there the factor $|q| \operatorname{deg}(Q)$ appears as the leading coefficient of $\left|Q^{\prime}\right|$.

If $\Delta_{j} \neq 0$, we have $m_{j}+j \notin I$, so that

$$
\left|\left(f^{\Delta_{j}}\right)^{\prime}\left(f^{m_{j}+j}\left(z_{0}\right)\right)\right| \geq\left|f^{\prime}\left(f^{m_{j}+j}\left(z_{0}\right)\right)\right| \geq \frac{R-\max _{s \in A}|s|}{2}\left|Q^{\prime}\left(f^{m_{j}+j}\left(z_{0}\right)\right)\right| .
$$

This implies the same conclusion as above with a factor which is larger than $|q| \operatorname{deg}(Q)$ for $R$ large enough.

Finally, due to the definition of $m_{j}$, for the last $j$ in the product we have $j+m_{j}=\alpha(n)$ so that this factor cancels out with the denominator in front of the product up to $\left|f^{\alpha(n)}\left(z_{0}\right)^{\delta / 4}\right|$ and we get

$$
\begin{aligned}
\frac{\left|\left(f^{\alpha(n)}\right)^{\prime}\left(z_{0}\right)\right|}{\left|f^{\alpha(n)}\left(z_{0}\right)\right|^{1-\operatorname{deg}(Q)+2 \delta} \geq} & \left|f^{\alpha(n)}\left(z_{0}\right)\right|^{\delta / 4}\left|\left(f^{j_{0}}\right)^{\prime}\left(z_{0}\right)\right|\left|Q^{\prime}\left(f^{j_{0}}\left(z_{0}\right)\right)\right| \\
& \times \prod_{\alpha(n)-m_{j}>j \in I} \frac{|q| \operatorname{deg}(Q)}{2}\left|f^{m_{j}+j}\left(z_{0}\right)\right|^{9 \delta / 4},
\end{aligned}
$$

which tends to infinity as $n \rightarrow \infty$.

Proof of Theorem 1.3. From the assumptions on the singular orbits it follows that $P(f)^{\prime} \cap J(f)=\emptyset$. An indifferent periodic point in the Julia set must be an accumulation point of $P(f)$. This is a well known fact, whose proof may be found in [20], where it is stated for rational functions. However only minor changes are necessary for the transcendental case.

Therefore all periodic points in $J(f)$ are repelling. Due to Theorem 3.1, we have $\omega(z) \subset P(f)$ for almost every $z \in J(f)$. If $O^{+}(z)$ accumulates at a repelling periodic point, $\omega(z)$ also accumulates at this point. This follows from the fact that $O^{+}(z)$ accumulates at every compact annulus $\left\{z: r \leq\left|z-z_{0}\right| \leq 2\left|\left(f^{p}\right)^{\prime}\left(z_{0}\right)\right| r\right\}$ if $r>0$ is small enough and $p$ is the period of the repelling periodic point $z_{0}$. Thus for almost every $z \in J(f)$ we have $\omega(z) \subset \overline{O^{+}(B)}$ if $B$ is the set of singularities that escape exponentially. Now Lemma 4.4 implies that the set of points that accumulate at no asymptotic values has measure zero. This concludes the proof for the inclusion $\omega(z) \supset A$.

For the other inclusion we assume that there exists $s \in B \backslash \overline{O^{+}(A)}$ such that $X^{\prime}:=\left\{z \in J(f): s \in \omega(z) \subset \overline{O^{+}(B)}\right\}$ has positive measure. Then the whole proof of Lemma 4.4 works identically, with $X^{\prime}$ instead of $X$, the only difference being that at the point where Lemma 4.3 is used we now use the measure estimate of Theorem 3.1. Since $O^{+}(T(f))$ is disjoint from $O^{+}\left(X^{\prime}\right)$, we find again that $X^{\prime}$ contains no density point, contradicting the assumption of positive measure. More precisely, instead of using the family $\mathcal{F}$ from Lemma 4.3 to see that the density of $\Gamma$ in 
$B_{n}:=B\left(f^{\alpha(n)}\left(z_{0}\right),\left|f^{\alpha(n)}\left(z_{0}\right)\right|^{1-\operatorname{deg}(Q)+2 \delta}\right)$ is bounded below, we use the family $\mathcal{S}$ from the proof for meas $(F(f))<\infty$ of Theorem 1.2 to see that the density of $T(f):=\left\{z: \omega(z) \subset \overline{O^{+}(A)}\right\}$ in $B_{n}$ is bounded below. We recall that the family $\mathcal{S}$ was a family of squares in $\{z: \operatorname{dist}(z, \mathbb{C} \backslash G) \geq$ $\left.|z|^{1-\operatorname{deg}(Q)+\delta}\right\}$ covering the set $\left\{z: \operatorname{dist}(z, \mathbb{C} \backslash G) \geq 2|z|^{1-\operatorname{deg}(Q)+\delta}\right\}$, such that for all $S \in \mathcal{S}$ we have $\sup _{z \in S}|z|^{1-\operatorname{deg}(Q)} \leq \operatorname{diam}(S) \leq 4 \sup _{z \in S}|z|^{1-\operatorname{deg}(Q)}$. The density of $T(f)$ in any $B_{n} \supset S \in \mathcal{S}$ is, due to Theorem 3.1, very close to 1 for large $n$. In particular, it is bounded below by some positive constant $c$. The choice of the diameter of $B_{n}$ implies that the density of $\left\{z: \operatorname{dist}(z, \mathbb{C} \backslash G) \leq 2|z|^{1-\operatorname{deg}(Q)+\delta}\right\}$ tends to zero as $n \rightarrow \infty$. The same is true for the union of those squares in $\mathcal{S}$ that intersect the boundary of $B_{n}$. This gives the estimate needed to proceed with the proof of Lemma 4.4.

Proof of Theorem 1.5. If all asymptotic values escape exponentially we can apply Theorem 1.2 and obtain a set of positive measure whose orbits accumulate only at the orbits of the asymptotic values and the point at infinity. In particular, the function is not recurrent. We now assume that the set of pre-periodic asymptotic values is non-empty and case (ii) of Theorem 1.1 holds. Since $P(f)^{\prime} \cap J(f)=\emptyset$, there are again no indifferent periodic points. From Lemma 4.4 we know that the orbit of almost every $z \in J(f)$ accumulates at least at one point in $P(f)$ which does not escape exponentially and thus has to be pre-periodic. By continuity, $O^{+}(z)$ accumulates at a repelling periodic point. As above this implies that $\omega(z)$ accumulates at this repelling periodic point. Since $P(f)$ has no cluster points in $\mathbb{C}$, this is a contradiction. Thus (i) of Theorem 1.1 is satisfied and $f$ is recurrent and ergodic on $J(f)=\mathbb{C}$.

The assumption that no critical point is periodic is no real restriction, since this case is trivial in terms of measurable dynamics. If this assumption is not satisfied the Fatou set obviously contains a Böttcher domain, so that $f$ is not recurrent and case (ii) of Theorem 1.1 holds.

5. Other applications. As mentioned in Remark 3.2, one can use Theorem 3.1 to obtain positive measure for the escaping set $I(f)$. As an example one can consider the following family containing the sine and cosine family, for which this result was proved by C. McMullen in [18].

TheOREM 5.1. Let $f(z):=P(z) \exp (Q(z))+\widetilde{P}(z) \exp (\widetilde{Q}(z))$, where $P, \widetilde{P}, Q$, and $\widetilde{Q}$ are polynomials with $\operatorname{deg}(P) \geq 0, \operatorname{deg}(\widetilde{P}) \geq 0$ and with $n:=\operatorname{deg}(\widetilde{Q})=\operatorname{deg}(Q)>0$ such that the arguments of their nth coefficients $q, \widetilde{q}$ differ by some odd multiple of $\pi / n$. Then the measure of $I(f)$ is positive. If $\widetilde{Q}=-Q$ and $n \geq 3$ then meas $(\mathbb{C} \backslash I(f))<\infty$. 
Sketch of proof. With the same arguments as in the proof of Theorem 1.2, one can show that for $0<\delta<\beta<1,-1<\delta_{1}<n-1<\delta_{2}$, $M$ large enough, $A:=\emptyset$ and

$$
G:=\left\{z:\left|\arg (z)-\frac{(2 k+1) \pi-2 \arg (q)}{2 n}\right| \leq|z|^{\delta-1}\right\}
$$

conditions (a) and (b) of Theorem 3.1 are satisfied, while condition (c) is trivial. The theorem implies the first part. The second part follows as in the proof of Theorem 1.2 by choosing $1-n<\delta<\beta<-1$.

As an example we consider the function

$$
f(z):=\exp \left(z^{3}\right)-\exp \left(-z^{3}\right) .
$$

Its Fatou set is not empty, since it contains a super-attractive basin around zero, and the theorem above gives $0<\operatorname{meas}(\mathbb{C} \backslash I(f))<\infty$. In Figure 6 the Fatou set is black. The picture shows the part of the plane given by $\{z:|\operatorname{Re}(z)| \leq 2,|\operatorname{Im}(z)| \leq 2\}$.

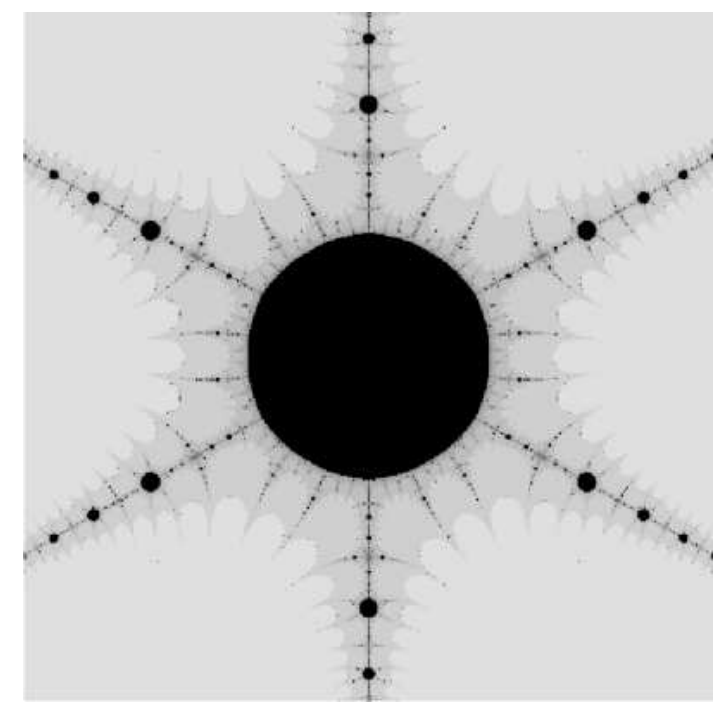

Fig. 6. The Fatou set of $f(z)=\exp \left(z^{3}\right)-\exp \left(-z^{3}\right)$

The functions discussed in the previous chapter have rational Schwarzian derivative $S(f):=f^{\prime \prime \prime} / f^{\prime}-\frac{3}{2}\left(f^{\prime \prime} / f^{\prime}\right)^{2}$. Much is known about functions whose Schwarzian derivative is a polynomial. The asymptotic behavior of functions with this property has already been studied by E. Hille [13] and R. Nevanlinna [21]. Most results carry over to the rational case, which was studied by G. Elfving [9]. It is easy to see that a critical point of $f$ is a 
pole of $S(f)$. Thus functions with a rational Schwarzian derivative have only finitely many critical points. If $S(f)(z)=c z^{n}(1+o(1))$ as $z \rightarrow \infty$ with $c \neq 0$ and $n \geq 0$, there are $n+2$ so-called critical rays defined by $\arg z=\phi$ with $\arg c+(n+2) \phi=0(\bmod 2 \pi)$. It turns out that these divide the complex plane into $n+2$ sectors in which the asymptotic behavior of $f$ is well known. If $z$ tends to infinity in a non-critical direction, $f$ tends to an asymptotic value, which is the same for all directions inside the same sector. Thus $f$ has only finitely many asymptotic values. Similarly to the proof of Theorem 1.2 one can show that conditions (a) and (b) of Theorem 3.1 are satisfied. If one of these asymptotic values happens to be $\infty$, points and also asymptotic values may escape exponentially inside the corresponding sector satisfying condition (c). However, these functions may have infinitely many poles, so that points can also escape exponentially, "jumping from pole to pole", without satisfying condition (c) of Theorem 3.1. The poles are, however, contained in small neighborhoods around these critical rays. Thus we can formulate another more geometric condition in order to guarantee condition (c). More precisely, we get the following.

THEOREM 5.2. Let $f$ be a meromorphic function with rational Schwarzian derivative behaving at infinity as $c z^{n}(1+o(1))$ with $c \neq 0$ and $n \geq-1$. Suppose that all asymptotic values $s$ tend to $\infty$ under iteration and there exists some $\varepsilon>0$ such that

$$
\left|\arg \left(f^{m}(s)\right)-\frac{2 \pi k+\arg (c)}{n+2}\right| \geq\left|f^{m}(s)\right|^{\varepsilon-(n+2) / 2}
$$

for almost all $m \in \mathbb{N}$ and all $k \in\{0,1, \ldots, n+1\}$. Then meas $(J(f))>0$ and $\omega(z) \subset P(f)$ for almost every $z \in J(f)$. If $n \geq 3$ then meas $(F(f))<\infty$.

Sketch of proof. The principle is exactly as in the proof of Theorem 1.2. First one has to check that the properties of Theorem 3.1 are satisfied. This gives us measure estimates of $T(f)$ that imply case (ii) of Theorem 1.1. We obtain $T(f) \subset J(f)$ again from the absence of Baker and wandering domains, which once more follows from the finiteness of $\operatorname{sing}\left(f^{-1}\right)$ (see Remark 3.2). For meromorphic functions with polynomial Schwarzian derivative this has also been shown by R. L. Devaney and L. Keen in $[8]$.

To check the properties we briefly summarize how to obtain estimates of the asymptotic behavior of $f$. We refer to the post-graduate notes of Jim Langley [15] for more details. It is easy to see that functions with the rational Schwarzian derivative $S(f)=2 A$ coincide with quotients $f_{1} / f_{2}$ of two linearly independent solutions of the differential equation $f_{i}^{\prime \prime}+A f_{i}=0$. Moreover, the asymptotic behavior of these solutions is well known. For a 
critical ray with argument $\phi$ and $R_{0}>0$ large we define

$$
Z(z):=\int_{2 R_{0} e^{i \phi}}^{z} A(t)^{1 / 2} d t=\frac{2 c^{1 / 2}}{n+2} z^{(n+2) / 2}\left(1+O\left(\frac{\ln |z|}{|z|}\right)\right) \quad \text { as } z \rightarrow \infty
$$

in the set $\left\{z: R_{0} \leq|z|,|\arg z-\phi| \leq 2 \pi /(n+2)\right\}$. Then it is easy to see that for $\delta^{\prime}>0$ and $R_{1}$ large enough, the change of coordinates $Z$ is univalent in $S_{1}:=\left\{z:|z| \geq R_{1},|\arg (z)-\phi|<2 \pi /(n+2)-\delta^{\prime}\right\}$. We use the Liouville transformation and consider $W_{i}(Z)=A(z)^{1 / 4} f_{i}(z)$, for which we get the differential equation

$$
\frac{\partial^{2} W_{i}}{\partial Z^{2}}+\left(1-F_{0}(Z)\right) W_{i}=0
$$

with $F_{0}(Z):=A^{\prime \prime}(z) / 4 A(z)^{2}-5 A^{\prime}(z)^{2} / 16 A(z)^{3}$. This equation has been integrated asymptotically by Hille [13] and his method has been used by many others afterwards. The following theorem and remark may be found explicitly in [15].

Theorem 5.3 (Hille, Langley). Let $c^{\prime}>0$ and $0<\varepsilon^{\prime}<\pi$. Then there exists $d^{\prime}>0$, depending only on $c$ and $\varepsilon^{\prime}$, with the following properties. Suppose that $F$ is analytic, with $|F(z)| \leq c^{\prime}|z|^{-2}$, in

$$
\Omega:=\left\{z: 1 \leq R_{0} \leq|z| \leq R_{1}<\infty,|\arg z| \leq \pi-\varepsilon^{\prime}\right\}
$$

Then the equation

$$
\omega^{\prime \prime}+(1-F(z)) \omega=0
$$

has two linearly independent solutions $U, V$ satisfying

$$
\begin{array}{ll}
U(z)=\exp (-i z)\left(1+\delta_{1}(z)\right), & U^{\prime}(z)=-i \exp (-i z)\left(1+\delta_{2}(z)\right), \\
V(z)=\exp (i z)\left(1+\delta_{3}(z)\right), & V^{\prime}(z)=i \exp (i z)\left(1+\delta_{4}(z)\right),
\end{array}
$$

such that $\left|\delta_{i}(z)\right| \leq d^{\prime}|z|^{-1}$ for $z \in \Omega \backslash\{z: \operatorname{Re}(z)<0,|\operatorname{Im}(z)|<R\}$.

REMARK. $\Omega$ may be replaced by

$$
\Omega^{\prime}:=\left\{z: 1 \leq R_{0} \leq|z| \leq R_{1}<\infty,|\arg z-\pi| \leq \pi-\varepsilon^{\prime}\right\}
$$

and also by the unbounded region

$$
\Omega^{\prime \prime}:=\left\{z: 1 \leq R_{0} \leq|z|<\infty,|\arg z| \leq \pi-\varepsilon^{\prime}\right\} .
$$

To see this, we take a sequence $R_{k} \rightarrow \infty$ and obtain solutions $U_{k}, V_{k}$ with uniformly bounded $\delta_{i, k}$ in $\Omega_{k}$, where $\Omega_{k}$ is $\Omega$ with $R_{1}$ replaced by $R_{k}$. Therefore both form a normal family, and a subsequence of $U_{k}, V_{k}$ converges in $\Omega^{\prime \prime}=\bigcup_{k \in \mathbb{N}} \Omega_{k}$. 
Thus for every $j \in\{1, . ., n+2\}$ and every critical ray with argument $\phi_{j}$ there are constants $a_{j}, b_{j}, c_{j}, d_{j} \in \mathbb{C}$ and $U_{j}, V_{j}$ such that

$$
\begin{gathered}
f(z)=\frac{a_{j} U_{j}(Z)+b_{j} V_{j}(Z)}{c_{j} U_{j}(Z)+d_{j} V_{j}(Z)} \\
V_{j}(z)=\exp \left(\frac{2 i c^{1 / 2}}{n+2} z^{(n+2) / 2}\left(1+O\left(\frac{\ln |z|}{|z|}\right)\right)\right)\left(1+O\left(|z|^{-1 / 2}\right)\right), \\
U_{j}(z)=\exp \left(\frac{-2 i c^{1 / 2}}{n+2} z^{(n+2) / 2}\left(1+O\left(\frac{\ln |z|}{|z|}\right)\right)\right)\left(1+O\left(|z|^{-1 / 2}\right)\right)
\end{gathered}
$$

as $z \rightarrow \infty$ in

$$
S_{j}:=\left\{z: 1 \leq R_{0} \leq|z|,\left|\arg (z)-\phi_{j}\right|<\frac{2 \pi}{n+2}-\delta\right\} .
$$

Thus $f$ tends to $a_{j} / c_{j}$ in the half-sector $S_{j}^{+}:=\left\{z \in S_{j}: \arg (z)>\phi_{j} \mid\right\}$ and to $b_{j} / d_{j}$ in the other half-sector $S_{j}^{-}:=\left\{z \in S_{j}: \arg (z)<\phi_{j}\right\}$. If $c_{j}$ or $d_{j}$ happen to be zero, while $a_{j}$ or $b_{j}$ are not, we obtain a sector, on which $f$ tends to $\infty$, such that points may escape exponentially in this sector. We get a similar estimate for the derivative, so that we can prove with similar arguments to those in the proof of Theorem 1.2 that $f$ satisfies the conditions of Theorem 3.1 for $0<\delta<\varepsilon, \delta-n / 2<\beta<1,-1<\delta_{1}<n / 2<\delta_{2}, M$ large enough, and $G:=\bigcup_{1<j<n+2}\left\{z \in S_{j}:\left|\operatorname{Im}\left(Z_{j}(z)\right)\right| \geq\left|Z_{j}(z)\right|^{2 \delta /(n+2)}\right\}$, where $Z_{j}$ is the above change of coordinates $Z$ for the sector $S_{j}$. If $n \geq 3$, we can choose $\delta<(n-2) / 2$. Then the proof of meas $(F(f))<\infty$ goes just as in Theorem 1.2.

\section{References}

[1] I. N. Baker, Wandering domains in the iteration of entire functions, Proc. London Math. Soc. 49 (1984), 563-576.

[2] - Infinite limits in the iteration of entire functions, Ergodic Theory Dynam. Systems 8 (1988), 503-507.

[3] I. N. Baker, J. Kotus and Y. Lü, Iterates of meromorphic functions III: Preperiodic domians, ibid. 11 (1991), 603-618.

[4] - - - - Iterates of meromorphic functions IV: Critically finite functions, Results Math. 22 (1992), 651-656.

[5] H. Bock, On the dynamics of entire functions on the Julia set, ibid. 30 (1996), $16-20$.

[6] -, Über das Iterationsverhalten meromorpher Funktionen auf der Juliamenge, Dissertation, Aachener Beiträge zur Mathematik 23 (1998).

[7] J. Conway, Functions of One Complex Variable 1, Springer, 1978.

[8] R. L. Devaney and L. Keen, Dynamics of meromorphic maps: Maps with polynomial Schwarzian derivative, Ann. Sci. École Norm. Sup. 22 (1989), 55-79.

[9] G. Elfving, Über eine Klasse von Riemannschen Flächen und ihre Uniformisierung, Acta Soc. Sci. Fenn. 2 (1934). 
[10] A. Eremenko and M. Lyubich, Dynamical properties of some classes of entire functions, Ann. Inst. Fourier (Grenoble) 42 (1992), 889-1019.

[11] L. R. Goldberg and L. Keen, A finiteness theorem for a dynamical class of entire functions, Ergodic Theory Dynam. Systems 6 (1986), 183-192.

[12] J.-M. Hemke, Typische Orbits der Exponentialfamilie, Diplomarbeit, 2002.

[13] E. Hille, On the zeros of the functions of the parabolic cylinder, Ark. Mat. 18 (1925).

[14] L. Keen and J. Kotus, Ergodicity of some classes of meromorphic functions, Ann. Acad. Sci. Fenn. 24 (1999), 133-145.

[15] J. Langley, Postgraduate notes on complex analysis, on J. Langley's website: www. maths.nott.ac.uk/personal/jkl/pg1.pdf, 40-50.

[16] M. Lyubich, On typical behaviour of the trajectories of a rational mapping on the sphere, Soviet Math. Dokl. 27 (1983), 22-25.

[17] - The measurable dynamics of the exponential, Sibirsk. Mat. Zh. 28 (1987), no. 5 111-127 (in Russian).

[18] C. McMullen, Area and Hausdorff dimension of Julia sets of entire functions, Trans. Amer. Math. Soc. 300 (1987), 329-342.

[19] —, Complex Dynamics and Renormalization, Princeton Univ. Press, 1994.

[20] J. Milnor, Dynamics in One Complex Variable: Introductory Lectures, Vieweg, Braunschweig, 2000.

[21] R. Nevanlinna, Über Riemannsche Flächen mit endlich vielen Windungspunkten, Acta Math. B 58 (1932), 295-373.

[22] —, Eindeutige analytische Funktionen, Grundlehren Math. Wiss. 46, Springer, 1936, 278-292.

[23] C. Pommerenke, Univalent Functions, Vandenhoeck und Ruprecht, Göttingen, 1975.

[24] M. Rees, The exponential map is not recurrent, Math. Z. 191 (1986), 593-598.

[25] P. J. Rippon and G. M. Stallard, Iteration of a class of hyperbolic meromorphic functions, Proc. Amer. Math. Soc. 127 (1999), 3251-3258.

[26] H. Schubert, Über das Maß der Fatoumenge trigonometrischer Funktionen, Diplomarbeit, 2003.

[27] B. Skorulski, Non-ergodic maps of the tangent family, Indag. Math. N.S. 14 (2003), 103-118.

[28] M. Urbański and A. Zdunik, Geometry and ergodic theory of non-hyperbolic exponential maps, preprint, 2004.

Mathematisches Institut der

Christian-Albrechts-Universität zu Kiel

Ludwig-Meyn-Str. 4, 24118 Kiel, Germany

E-mail: hemke@math.uni-kiel.de

Received 1 March 2005;

in revised form 8 July 2005 